TRANSACTIONS OF THE

AMERICAN MATHEMATICAL SOCIETY

Volume 355, Number 11, Pages 4427-4450

S 0002-9947(03)03177-5

Article electronically published on July 2, 2003

\title{
EXAMPLES FOR THE MOD $p$ MOTIVIC COHOMOLOGY OF CLASSIFYING SPACES
}

\author{
NOBUAKI YAGITA
}

\begin{abstract}
Let $B G$ be the classifying space of a compact Lie group $G$. Some examples of computations of the motivic cohomology $H^{*, *}(B G ; \mathbb{Z} / p)$ are given, by comparing with $H^{*}(B G ; \mathbb{Z} / p), C H^{*}(B G)$ and $B P^{*}(B G)$.
\end{abstract}

\section{INTRODUCTION}

Let $p$ be a prime number and $k$ a subfield of the complex number field $\mathbb{C}$. Let $k$ contain a primitive $p$-th root of unity. Given a scheme $X$ of finite type over $k$, the mod $p$ motivic cohomology $H^{*, *}(X ; \mathbb{Z} / p)=\bigoplus_{m, n} H^{m, n}(X ; \mathbb{Z} / p)$ has been defined by Suslin and Voevodsky ([Vo1], [Vo2]). When $X$ is smooth, the subring $H^{2 *, *}(X ; \mathbb{Z} / p)=\bigoplus_{n} H^{2 n, n}(X ; \mathbb{Z} / p)$ is identified with the classical mod $p$ Chow ring $C H^{*}(X) / p$ of algebraic cyles on $X$.

The inclusion $t_{\mathbb{C}}: k \subset \mathbb{C}$ induces a natural transformation (realization map) $t_{\mathbb{C}}^{m, n}: H^{m, n}(X ; \mathbb{Z} / p) \rightarrow H^{m}(X(\mathbb{C}) ; \mathbb{Z} / p)$, where $X(\mathbb{C})$ is the complex variety of $\mathbb{C}$-valued points of $X$. Let us write the coimage of $t_{\mathbb{C}}^{*, *}$ as

$$
h^{*, *}(X ; \mathbb{Z} / p)=\bigoplus_{m, n} H^{m, n}(X ; \mathbb{Z} / p) / \operatorname{Ker}\left(t_{\mathbb{C}}^{m, n}\right) .
$$

It is known that there is an element $\tau \in H^{0,1}(\operatorname{Spec}(k) ; \mathbb{Z} / p)$ with $t_{\mathbb{C}}^{*, *}(\tau)=1$. Then we have the bigraded $\mathbb{Z} / p[\tau]$-algebra monomorphism

$$
h^{*, *}(X ; \mathbb{Z} / p) \hookrightarrow H^{*}(X(\mathbb{C}) ; \mathbb{Z} / p) \otimes \mathbb{Z} / p\left[\tau, \tau^{-1}\right]
$$

where the bidegree of $x \in H^{n}(X(\mathbb{C}) ; \mathbb{Z} / p)$ is given by $(n, n)$. If $k=\mathbb{C}$ and the Beilinson-Lichtenbaum condition $\sqrt{ }$ o2 is satisfied for $p$, then we also have the injection $H^{*}(X(\mathbb{C}) ; \mathbb{Z} / p) \otimes \mathbb{Z} / p[\tau] \hookrightarrow h^{* * *}(X ; \mathbb{Z} / p)$.

When $x \in H^{m, n}(X ; \mathbb{Z} / p)$, define the weight of $x$ by $w(x)=2 n-m$. Clearly $w(x)=0$ if and only if $x \in C H^{*}(X) / p$. Voevodsky has extended the Steenrod algebra $A_{p}^{*}$ of cohomology operations to the case of motivic cohomology. Among them, we have the Milnor primitive operation

$$
Q_{i}: H^{*, *}(X ; \mathbb{Z} / p) \rightarrow H^{*+2 p^{i}-1, *+p^{i}-1}(X ; \mathbb{Z} / p),
$$

so that it is sent to the usual Milnor operation $Q_{i}$ by the realization map $t_{\mathbb{C}}^{*}$. Hence $w\left(Q_{i}\right)=-1$, and the $Q_{i}(0 \leq i)$ form an exterior algebra $\Lambda\left(Q_{0}, Q_{1}, \ldots\right) \subset A_{p}^{*}$ also for the motivic cohomology. To simplify the notation, let us write the exterior algebra $Q(n)=\Lambda\left(Q_{0}, \ldots, Q_{n}\right)$ for $n \geq 0$ and $Q(-1)=\mathbb{Z} / p$.

Received by the editors January 10, 2002.

2000 Mathematics Subject Classification. Primary 55P35, 57T25; Secondary 55R35, 57T05.

Key words and phrases. Motivic cohomology, Chow ring, BP-theory. 
In this paper we are mainly concerned with the following case. For $n \geq-1$, let $G_{n}$ be a $\mathbb{Z} / p$-module and $Q(n) G_{n}$ the free $Q(n)$-module generated by $G_{n}$. Moreover, the scheme $X$ satisfies the assumption that there is a $\mathbb{Z} / p$-module injection

$$
j_{\mathbb{C}}: H^{*}(X(\mathbb{C}) ; \mathbb{Z} / p) \hookrightarrow \bigoplus_{n=-1}^{\infty} Q(n) G_{n} \quad \text { with } j_{\mathbb{C}}^{-1}\left(Q_{0} \ldots Q_{n} G_{n}\right) \subset \operatorname{Im}\left(t_{\mathbb{C}}^{2 *, *}\right)
$$

such that $p_{n} j_{\mathbb{C}}: H^{*}(X(\mathbb{C}): \mathbb{Z} / p) \rightarrow Q(n) G_{n}$ is the $Q(n)$-module map and $p_{n}^{\prime} p_{n} j_{\mathbb{C}}:$ $H^{*}(X(\mathbb{C}): \mathbb{Z} / p) \rightarrow Q_{0} \ldots Q_{n-1} G_{n}$ is a surjection for each $n$, where $p_{n}: \bigoplus Q(n) G_{n} \rightarrow$ $Q(n) G_{n}$ and $p_{n}^{\prime}: Q(n) G_{n} \rightarrow Q_{0} \ldots Q_{n-1} G_{n}$ are the projections. (We do not assume a $Q(n)$-module structure on the right-hand side module in (1.3).)

We take the weight on the right-hand side by putting $w(x)=n+1$ for every $x \in G_{n}$ (simply write $w\left(G_{n}\right)=n+1$ ), so that $w\left(Q_{0} \ldots Q_{n} x\right)=0$. Then we get the injection of bigraded $\mathbb{Z} / p$-modules

$$
j: h^{*, *}(X ; \mathbb{Z} / p) \hookrightarrow \bigoplus_{n=-1}^{\infty} Q(n) G_{n} \otimes \mathbb{Z} / p[\tau]
$$

such that the composition $\left(p_{n} \otimes \mathbb{Z} / p[\tau]\right) j: h^{*, *}(X ; \mathbb{Z} / p) \rightarrow Q(n) G_{n} \otimes \mathbb{Z} / p[\tau]$ is the bigraded $Q(n)$-module map.

The above argument has its counterpart in the $B P$-theory of $X(\mathbb{C})$. As we know, $B P^{*}(-)$ is the cohomology theory with the coefficient ring $B P^{*}=\mathbb{Z}_{(p)}\left[v_{1}, v_{2}, \ldots\right]$, $\left|v_{i}\right|=-2\left(p^{i}-1\right)$. Let us write $B P^{*} /\left(p, v_{1}, \ldots, v_{m-1}\right)$ as $P(m)^{*}$. The AtiyahHirzebruch spectral sequence

$$
E_{2}^{*, *}=H^{*}(X(\mathbb{C})) \otimes B P^{*} \Longrightarrow B P^{*}(X(\mathbb{C}))
$$

has the differential

$$
d_{2 p^{i}-1}(x)=Q_{i}(x) \otimes v_{i} \quad \bmod \left(M_{i}\right),
$$

where $M_{i}$ is the ideal of $E_{2 p^{i}-1}^{* *}$ generated by elements in $\left(p, v_{1}, \ldots, v_{i-1}\right) E_{2}^{*, *}$ We assume here that nonzero differentials are all of the form $(1.5)$ and that $H^{*}(X(\mathbb{C}))$ has no higher $p$-torsion. Then we easily see that (1.3) implies

$$
E_{\infty}^{* * *} \cong \bigoplus_{n=-1}^{\infty} P(n+1)^{*} \tilde{G}_{n} \oplus B \quad \text { with } \quad \tilde{G}=Q_{0} \ldots Q_{n} G_{n}
$$

where $P(n+1)^{*} \tilde{G}_{n}$ is the free $P(n+1)^{*}$-module generated by elements in $\tilde{G}_{n}$ and $B$ is the $B P^{*}$-submodule of $E_{\infty}^{*, *}$ of generators in $\operatorname{Ideal}\left(p, v_{1}, \ldots\right) E_{2}^{*, *}$. Conversely, by the same assumption, if $\tilde{G}_{n} \subset \operatorname{Im}\left(t_{\mathbb{C}}^{2 * * *}\right)$, then the isomorphism (1.6) implies the existence of the injections $j_{\mathbb{C}}$ in (1.3) and so $j$ in (1.4).

Let $\rho: B P(X(\mathbb{C})) \otimes_{B P^{*}} \mathbb{Z} / p \rightarrow H^{*}(X(\mathbb{C}) ; \mathbb{Z} / p)$ be the Thom map. Then (1.6) and $\tilde{G}_{n} \subset \operatorname{Im}\left(t_{\mathbb{C}}^{2 * *}\right)$ imply that

$$
\operatorname{Im}\left(t_{\mathbb{C}}^{2 * * *}\right)=\operatorname{Im}(\rho) \cong \bigoplus_{n=-1}^{\infty} \tilde{G}_{n} \subset B P^{*}(X(\mathbb{C})) \otimes_{B P^{*}} \mathbb{Z} / p
$$

More generally, B. Totaro [To1], To2] constructed the modified cycle map

$$
\tilde{c l}^{*}: C H^{*}(X) / p \rightarrow B P^{*}(X(\mathbb{C})) \otimes_{B P^{*}} \mathbb{Z} / p
$$

in such a way that the composition $\rho \tilde{c l}^{*}$ is the realization map $t_{\mathbb{C}}^{2 * * *}$. If a $B P^{*}$ module generator of $B$ in (1.6) is represented by transfer of a Chern class, then 
this element gives a nonzero element in $\operatorname{Ker}\left(t_{\mathbb{C}}^{2 * *}\right)$ by the modified cycle map $\tilde{c l}^{*}$. Using this argument, Totaro found nonzero elements in $\operatorname{Ker}\left(t_{\mathbb{C}}^{2 * *}\right)$ when $X$ is the classifying space $B S O(4)$.

The motivic cohomology of the classifying space is defined as follows. Let $G$ be a linear algebraic group over $k$. Let $V$ be a representation of $G$ such that $G$ acts freely on $V-S$ for some closed subset $S$. Then $(V-S) / G$ exists as a quasi-projective variety over $k$. Following Totaro To1] and Voevodsky, define

$$
H^{*, *}(B G ; \mathbb{Z} / p)=\lim _{\operatorname{dim}(V), \operatorname{codim}(S) \rightarrow \infty} H^{*, *}((V-S) / G ; \mathbb{Z} / p) .
$$

The topological space $B G(\mathbb{C})=\lim ((V-S) / G)(\mathbb{C})$ is the usual classifying space $B G$. Hence we write the $\mathbb{C}$-value points $B G(\mathbb{C})$ simply as $B G$.

We will show that the isomorphism (1.6) is satisfied when $X=B G$ for the following cases: $O(n), S O(4), D_{8}, G_{2}, \operatorname{Spin}(7)$ for $p=2, P G L_{3}, F_{4}$ for $p=3$ and the extraspecial $p$-group $p_{+}^{1+2}$ of order $p^{3}$ and of exponent $p$ for odd primes. (However note that $H^{*}\left(B p_{+}^{1+2}\right)$ has $p^{2}$-torsion.)

Hence we will prove (1.4) for these $B G$. Moreover, when $k=\mathbb{C}$ and $G=O(3)$ for $p=2, P G L_{3}$ for $p=3, p_{+}^{1+2}$ and $(\mathbb{Z} / p)^{n}$ for all primes, we will show that

$$
h^{*, *}(B G ; \mathbb{Z} / p) \cong \bigoplus Q(n) G_{n} \otimes \mathbb{Z} / p[\tau]
$$

S. Wilson RWY first constructed the decomposition (1.3) so that $j_{\mathbb{C}}$ is an isomorphism for $X=B O(n)$, and next computed $B P^{*}(B O(n))$. However, it is unknown whether $j$ in (1.4) is an isomorphism or not for $X=B O(n), n \geq 4$.

The contents of this paper are as follows. The aim of $\S \S 2$ and 3 is a short introduction to motivic cohomology for algebraic topologists unfamiliar with it. In these sections, we concentrate on the computation of $H^{*}\left(B(\mathbb{Z} / p)^{n} ; \mathbb{Z} / p\right)$. In $\S 4$, we deal with the study of $h^{*, *}(X ; \mathbb{Z} / p)$, making no use of $B P^{*}(B G)$ but Milnor's operation $Q_{i}$ instead. In $\oint 5$, we give an account of $h^{*, *}(B G ; \mathbb{Z} / p)$ expressed in term of $B P^{*}(B G)$. Also in this section we give some results on $\operatorname{Ker}\left(t_{\mathbb{C}}^{*, *}\right)$. The motivic cohomology of the Eilenberg-MacLane space $K(\mathbb{Z} / p(n), n)$ is studied in $\S 6$. In $\S 7$, we give some comments on algebraic cobordism theory and algebraic $B P$-theory.

Finally, we want to express our deep gratitude to Professors Burt Totaro, Tokushi Nakamura, and Toshio Kawashima for their kind help in correcting many errors in the first draft of this paper.

\section{Chow ring, Milnor K-theory, Étale cohomology}

We use the category $S p c$ of (algebraic) spaces, along with schemes $A$, their quotients $A_{1} / A_{2}$ and $\operatorname{colim}\left(A_{\alpha}\right)$, all defined by Voevodsky [Vo2], [MoVo]. Here schemes are defined over a field $k$ with $c h(k)=0$. The motivic cohomology is the double indexed cohomology defined by Suslin and Voevodsky, directly related with the Chow ring and Milnor $K$-theory.

$(\mathrm{CH})$ For a smooth scheme $X$ we have $H^{2 n, n}(X) \cong C H^{n}(X)$, the classical Chow group of codim $n$ cycles on $X$.

$(\mathrm{MK}) H^{n, n}(\operatorname{Spec}(k)) \cong K_{n}^{M}(k)$, the Milnor $K$-group for the field $k$.

For a smooth variety $X$ with $\operatorname{dim}(X)=n$, the Chow ring is the sum $C H^{*}(X)=$ $\bigoplus_{i} C H^{i}(X)$, where

$$
C H^{i}(X)=\{(n-i) \text { cycles in } X\} /(\text { rational equivalence }) .
$$


Here the rational equivalence $a \equiv b$ is defined if there is a codimension $i$ subvariety $W$ in $X \times \mathbb{P}^{1}$ such that $a=p_{*} f^{*}(0)$ and $b=p_{*} f^{*}(1)$, where $\mathbb{P}^{1}$ is the projective line and $p$ (resp. $f$ ) is the projection on the first (resp. second) factor.

For $k=\mathbb{C}$, if $X$ has a cellular decomposition, i.e., $X=X_{n} \supset X_{n-1} \supset \ldots \supset X_{0}$ with $X_{i}-X_{i-1}=\bigcup \mathbb{A}^{n_{i j}}$, where $\mathbb{A}^{n_{i j}}$ is the affine space of dimension $n_{i j}$, then $C H^{*}(X) \cong H^{*}(X(\mathbb{C}))$, the singular cohomology theory of $\mathbb{C}$-rational points of $X$. For example, $C H^{*}\left(\mathbb{P}^{n}\right) \cong H^{*}\left(\mathbb{C P}^{n}\right)$ for projective spaces $\mathbb{P}^{n}$. Since $S p c$ contains colimit, we can consider the infinite projective space $\mathbb{P}^{\infty}=B \mathbb{G}_{m}$ and the infinite lens space $\lim _{n}\left(\mathbb{A}^{n}-\{0\} / \mathbb{Z} / p\right)=L_{p}^{\infty}=B \mathbb{Z} / p$. The Chow rings of classifying spaces of abelian groups are given in [To1]:

$$
C H^{*}\left(\mathbb{P}^{\infty}\right) \cong H^{2 *, *}\left(\mathbb{P}^{\infty}\right) \cong \mathbb{Z}[y], \quad C H^{*}(B \mathbb{Z} / p) \cong H^{2 *, *}(B \mathbb{Z} / p) \cong \mathbb{Z}[y] /(p y),
$$

with $\operatorname{deg}(y)=(2,1)$. For products of these spaces we have

$$
\begin{gathered}
C H^{*}\left(\left(\mathbb{P}^{\infty}\right)^{n}\right) \cong \mathbb{Z}\left[y_{1}, \ldots, y_{n}\right], \\
C H^{*}\left((B \mathbb{Z} / p)^{n}\right) \cong \mathbb{Z}\left[y_{1}, \ldots, y_{n}\right] /\left(p y_{1}, \ldots, p y_{n}\right) .
\end{gathered}
$$

Here note that $C H^{*}(X) \neq H^{\text {even }}(X(\mathbb{C}))$ for the last case. Even if $H^{*}(X(\mathbb{C}))$ is generated by even dimensional elements, there are cases that $C H^{*}(X) \neq H^{*}(X(\mathbb{C}))$, e.g., K3-surfaces have the cohomology $H^{2}(X(\mathbb{C})) \cong \mathbb{Z}^{22}$, but there is a K3-surface such that $C H^{1}(X) \cong \mathbb{Z}^{i}$ for each $1 \leq i \leq 20$.

Milnor $K$-theory is the graded ring $\bigoplus_{n} K_{n}^{M}(k)$ defined by $K_{n}^{M}(k)=\left(k^{*}\right)^{\otimes n} / J$, where the ideal $J$ is generated by elements $a \otimes(1-a)$ for $a \in k^{*}-\{1\}$. Here the addition of $k^{*}$ is given by the multiplication in the field $k$. Hence $K_{0}^{M}(k)=\mathbb{Z}$ and $K_{1}^{M}(k)=k^{*}$. Hilbert's Theorem 90, which essentially says that the Galois cohomology $H^{1}\left(G\left(k_{s} / k\right) ; k_{s}^{*}\right)=0$, implies the isomorphism $K_{1}^{M}(k) / p \cong k^{*} /\left(k^{*}\right)^{p} \cong$ $H^{1}\left(G\left(k_{s} / k\right) ; \mathbb{Z} / p\right)$ for $1 / p \in k$. Similarly we can define a map (the norm residue map) for any extension $F$ of $k$ of finite type,

$$
K_{n}^{M}(F) / p \rightarrow H^{n}\left(G\left(F_{s} / F\right) ; \mu_{p}^{\otimes n}\right)
$$

where $\mu_{p}^{\otimes n}$ is the discrete $G\left(F_{s} / F\right)$-module of $n$-th tensor power of the group of $p$-roots of 1 . The Bloch-Kato conjecture is that this map is an isomorphism for all field $k$, and the Milnor conjecture is its $p=2$ case. This conjecture is solved when $n=2$ by Merkurjev and Suslin [MeSu], and for $p=2$ by Voevodsky [Vo1].

Notice that $H^{n}\left(G\left(k_{s} / k\right) ; \mu_{p}^{\otimes n}\right) \cong H_{e t}^{n}\left(\operatorname{Spec}(k), \mu_{p}^{\otimes n}\right)$, the étale cohomology of the point. The étale cohomology $H_{e t}^{*}(X ; \mathbb{Z} / p)$ has the following properties:

(E.1) If $k$ contains a primitive $p$-th root of 1 , then there is the additive isomorphism

(E.2) For smooth $X$ over $k=\mathbb{C}$,

$$
H_{e t}^{m}\left(X, \mu_{p}^{\otimes n}\right) \cong H_{e t}^{m}(X ; \mathbb{Z} / p) .
$$

$$
H_{\text {et }}^{m}\left(X ; \mathbb{Z} / p^{N}\right) \cong H^{m}\left(X(\mathbb{C}) ; \mathbb{Z} / p^{N}\right) \text { for all } N \geq 1 .
$$

The last cohomology is the usual mod $p$ ordinary cohomology of $\mathbb{C}$-rational points of $X$. Of course $H_{e t}^{*}(\operatorname{Spec}(\mathbb{C}) ; \mathbb{Z} / p) \cong \mathbb{Z} / p$. It is known that

$$
K_{*}^{M}(\mathbb{R}) / 2 \cong H_{\text {et }}^{*}(\operatorname{Spec}(\mathbb{R}) ; \mathbb{Z} / 2) \cong \mathbb{Z} / 2[\rho]
$$

with $\operatorname{deg}(\rho)=1$ for the real number field $\mathbb{R}$. Let $F_{v}$ be a local field with residue field $k_{v}$ of $\operatorname{ch}\left(k_{v}\right) \neq 2$. Then $K_{*}^{M}\left(F_{v}\right) / 2 \cong H_{e t}^{*}\left(\operatorname{Spec}\left(F_{v}\right) ; \mathbb{Z} / 2\right) \cong \Lambda(\alpha, \beta)$ with $\operatorname{deg}(\alpha)=\operatorname{deg}(\beta)=1$. Thus we know that $\bigoplus_{m} H^{m, m}(p t ; \mathbb{Z} / 2)$ for these cases. 


\section{The REALizATION MAP}

In this section we consider the relation to the usual ordinary cohomology. Let $R$ be $\mathbb{Z}$ or $\mathbb{Z} / p$. The motivic cohomology has the following properties [Vo2].

(C1) $H^{*, *}(X ; R)$ is a bigraded ring natural in $X$.

(C2) When $k \subset \mathbb{C}$, there are maps (realization maps)

$$
t_{\mathbb{C}}^{m, n}: H^{m, n}(X ; R) \rightarrow H^{m}(X(\mathbb{C}) ; R)
$$

which sum up to $t_{\mathbb{C}}^{*, *}=\bigoplus_{m, n} t_{\mathbb{C}}^{m, n}$, the natural ring homomorphism.

(C3) There are the (Bockstein, reduced powers) operations

$$
\begin{gathered}
\beta: H^{*, *}(X ; \mathbb{Z} / p) \rightarrow H^{*+1, *}(X ; \mathbb{Z} / p), \\
P^{i}: H^{*, *}(X ; \mathbb{Z} / p) \rightarrow H^{*+2(p-1) i, *+(p-1) i}(X ; \mathbb{Z} / p),
\end{gathered}
$$

which commutes with the realization map $t_{\mathbb{C}}$ when $k \subset \mathbb{C}$.

(C4) For the projective space $\mathbb{P}^{n}$, there is an isomorphism

$$
H^{*, *}\left(X \times \mathbb{P}^{n} / \mathbb{P}^{n-1} ; R\right) \cong H^{*, *}(X ; R)\left\{1, y^{\prime}\right\}
$$

with $\operatorname{deg}\left(y^{\prime}\right)=(2 n, n)$ and $t_{\mathbb{C}}\left(y^{\prime}\right) \neq 0$ for $k \subset \mathbb{C}$.

We recall the Lichtenbaum motivic cohomology $\mathrm{Vo}_{\mathrm{O}}$. Lichtenbaum defined the similar cohomology $H_{L}^{*, *}(X ; R)$ by using the étale topology, while $H^{*, *}(X ; R)$ is defined using the Nisnevich topology. Since Nisnevich covers are restricted étale covers, there is the natural map $H^{*, *}(X ; R) \rightarrow H_{L}^{*, *}(X ; R)$. We say that the $B(n, p)$ condition holds if

$$
H^{m, n}\left(X ; Z_{(p)}\right) \cong H_{L}^{m, n}\left(X ; Z_{(p)}\right) \text { for all } m \leq n+1
$$

and all smooth $X$. The Beilinson-Lichtenbaum conjecture is that $B(n, p)$ holds for all $n$ and $p$. It is known that the condition $B(n, p)$ is equivalent to the Bloch-Kato conjecture $(\mathrm{BK})$ for degree $n$ and prime $p$. Hence $B(n, p)$ holds for $n \leq 2$ or $p=2$. Moreover, Suslin and Voevodsky have proved

$$
H_{L}^{m, n}(X ; \mathbb{Z} / p) \cong H_{e t}^{m}\left(X ; \mu_{p}^{\otimes n}\right) .
$$

Now we compute $H^{*, *}(p t ; \mathbb{Z} / p)=H^{*, *}(\operatorname{Spec}(k) ; \mathbb{Z} / p)$. For a smooth $X$, the following dimensional condition is known:

(C5) For a smooth $X$, if $H^{m, n}(X ; R) \neq 0$, then

$$
m \leq n+\operatorname{dim}(X), \quad m \leq 2 n \text { and } m \geq 0 .
$$

For the rest of this paper, we assume that $k$ contains a primitive $p$-th root of 1 and $B(n, p)$ holds for all $n$, but $X=\operatorname{Spec}(k)$. Then

$$
H^{m, n}(p t ; \mathbb{Z} / p) \cong H_{e t}^{m}\left(p t ; \mu_{p}^{\otimes n}\right) \cong H_{e t}^{m}(p t ; \mathbb{Z} / p) \quad \text { if } m \leq n
$$

and $H^{m, n}(p t ; \mathbb{Z} / p) \cong 0$ for $m>n$. Let $\tau \in H^{0,1}(p t ; \mathbb{Z} / p)$ be the element corresponding to a generator of $H_{e t}^{0}\left(\operatorname{Spec}(k) ; \mu_{p}\right) \cong H_{e t}^{0}(\operatorname{Spec}(k) ; \mathbb{Z} / p)$. Then we get the isomorphism

$$
H^{*, *}(\operatorname{Spec}(k) ; \mathbb{Z} / p) \cong H_{e t}^{*}(\operatorname{Spec}(k) ; \mathbb{Z} / p) \otimes \mathbb{Z} / p[\tau]
$$

since $\tau: H_{e t}^{m}\left(p t ; \mu_{p}^{\otimes n}\right) \cong H_{e t}^{m}\left(p t ; \mu_{p}^{\otimes(n+1)}\right)$. In particular, for the real number field $\mathbb{R}$ and a local field $F_{v}$ with the residue field $k_{v}$ of $\operatorname{ch}\left(k_{v}\right) \neq 2$ we have

$$
\begin{gathered}
H^{*, *}(\operatorname{Spec}(\mathbb{R}) ; \mathbb{Z} / 2) \cong \mathbb{Z} / 2[\rho, \tau] \quad \text { with } \operatorname{deg}(\rho)=(1,1), \\
H^{*, *}\left(\operatorname{Spec}\left(F_{v}\right) ; \mathbb{Z} / 2\right) \cong \mathbb{Z} / 2[\tau] \otimes \Lambda(\alpha, \beta) \quad \text { with } \operatorname{deg}(\alpha)=\operatorname{deg}(\beta)=(1,1) .
\end{gathered}
$$


For $k=\mathbb{C}$, we know that $K_{n}^{M}(\mathbb{C}) / p \cong 0$ for $n>0$, and hence

$$
H^{*, *}(\operatorname{Spec}(\mathbb{C}) ; \mathbb{Z} / p) \cong \mathbb{Z} / p[\tau] \quad \text { with } \operatorname{deg}(\tau)=(0,1) .
$$

When $k=\mathbb{C}$, if the $B(n, p)$ condition holds for $X$, then it is immediate that

$$
\left[\tau^{-1}\right] H^{*, *}(X ; \mathbb{Z} / p) \cong H^{*}(X(\mathbb{C}) ; \mathbb{Z} / p) \otimes \mathbb{Z} / p\left[\tau, \tau^{-1}\right],
$$

where the degree is defined by $\operatorname{deg}(x)=(m, m)$ if $x \in H^{m}(X(\mathbb{C}) ; \mathbb{Z} / p)$.

Next we compute the cohomology of $\mathbb{P}^{\infty}$ and $B \mathbb{Z} / p$. For any (algebraic) map $f: X \rightarrow Y$ in the category $S p c$, we can construct the cofiber sequence

$$
X \rightarrow Y \rightarrow \text { cone }(f)=Y / X,
$$

which induces the long exact sequence (Voevodsky [Ve2])

$$
H^{*, *}(X ; R) \leftarrow H^{*, *}(Y ; R) \leftarrow H^{*, *}(Y / X: R) \leftarrow H^{*-1, *}(X ; R) .
$$

In particular, we get the Mayer-Vietoris, Gysin and blow-up long exact sequences.

By the cofiber sequence $\mathbb{P}^{n-1} \rightarrow \mathbb{P}^{n} \rightarrow \mathbb{P}^{n} / \mathbb{P}^{n-1}$ and (C4), we can inductively see that

$$
H^{*, *}\left(\mathbb{P}^{n} ; \mathbb{Z} / p\right) \cong H^{*, *}(p t ; \mathbb{Z} / p) \otimes \mathbb{Z} / p[y] /\left(y^{n+1}\right) \quad \text { with } \operatorname{deg}(y)=(2,1) .
$$

When $k=\mathbb{C}$, since $B(1, p)$ always holds, $H^{1,1}\left(L_{p}^{n} ; \mathbb{Z} / p\right) \cong H^{1}\left(L_{p}^{n} ; \mathbb{Z} / p\right)$. Hence there is an element $x^{\prime} \in H^{1,1}\left(L_{p}^{n} ; \mathbb{Z} / p\right)$ with $t_{\mathbb{C}}\left(x^{\prime}\right)=x \in H^{1}\left(L_{p}^{n} ; \mathbb{Z} / p\right)$. This also holds for general $k$ Vo3. The lens space is identified with the sphere bundle associated with the line bundle

$$
\left(\mathbb{A}^{n}-\{0\}\right) \times(\mathbb{A}-\{0\}) \mathbb{A} \rightarrow\left(\mathbb{A}^{n}-\{0\}\right) /(\mathbb{A}-\{0\})=\mathbb{P}^{n} .
$$

Here $\left(\mathbb{A}^{n}-\{0\}\right) \times{ }_{(\mathbb{A}-\{0\})} \mathbb{A}$ is the identification such that $\left(z_{i}, z\right) \sim\left(a^{-1} z_{i}, a^{p} z\right) \in$ $\left(\mathbb{A}^{n}-\{0\}\right) \times \mathbb{A}$ for $\left(z_{i}\right) \in \mathbb{A}^{n}, z \in \mathbb{A}, a \in \mathbb{A}-\{0\}$. Hence we get the cofibering $L_{p}^{n} \rightarrow \mathbb{P}^{n} \stackrel{\times p}{\longrightarrow} \mathbb{P}^{n}$. Thus we get the additive isomorphism $H^{*, *}\left(L_{p}^{n} ; \mathbb{Z} / p\right) \cong$ $H^{*, *}\left(\mathbb{P}^{n} ; \mathbb{Z} / p\right)\{1, x\}$. This induces the ring isomorphism for $p=$ odd

$$
H^{*, *}\left(L_{p}^{n} ; \mathbb{Z} / p\right) \cong \mathbb{Z} / p[y] /\left(y^{n+1}\right) \otimes \Lambda(x) \otimes H^{*, *}(p t ; \mathbb{Z} / p) \quad \text { with } \operatorname{deg}(x)=(1,1) .
$$

However, note that when $p=2$ we get $x^{2}=y \tau+x \rho$ [Vo3], where $\rho \in H^{1,1}(p t ; \mathbb{Z} / p) \cong$ $k^{*} / k^{2 *}$ represents -1 . (Hence $\rho=0$ when $\sqrt{-1} \in k^{*}$.) This is proved by the wellknown fact that $\{a, a\}=\{a,-1\}$ in the Milnor $K$-theory $K_{2}^{M}(k)$.

We say that a space $X$ satisfies the Künneth formula for a space $Y$ if

$$
H^{*, *}(X \times Y ; \mathbb{Z} / p) \cong H^{*, *}(X ; \mathbb{Z} / p) \otimes_{H^{*, *}(p t ; \mathbb{Z} / p)} H^{*, *}(Y ; \mathbb{Z} / p) .
$$

By the above cofiber sequences, we can easily see that $\mathbb{P}^{\infty}$ and $B \mathbb{Z} / p$ satisfy the Künneth formula for all spaces. In particular, we have the ring isomorphisms

$$
\begin{gathered}
H^{*, *}\left(\left(\mathbb{P}^{\infty}\right)^{n} ; \mathbb{Z} / p\right) \cong \mathbb{Z} / p\left[y_{1}, \ldots, y_{n}\right] \otimes H^{*, *}(p t ; \mathbb{Z} / p), \\
H^{*, *}\left((B \mathbb{Z} / p)^{n} ; \mathbb{Z} / p\right) \cong \mathbb{Z} / p\left[y_{1}, \ldots, y_{n}\right] \otimes \Lambda\left(x_{1}, \ldots, x_{n}\right) \otimes H^{*, *}(p t ; \mathbb{Z} / p)
\end{gathered}
$$

(when $p=2, x_{i}^{2}=y_{i} \tau+x_{i} \rho$ ).

This fact is used to define the reduced power operation $P^{i}$ in (C3). Since a Sylow $p$-subgroup of the symmetric group $S_{p}$ of $p$ letters is isomorphic to $\mathbb{Z} / p$, we have the isomorphism

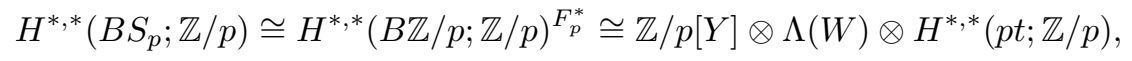


identifying $Y=y^{p-1}$ and $W=x y^{p-2}$. If $X$ is smooth (and suppose $p$ is odd, to simplify arguments), we can define the reduced powers (of Chow rings) as follows. Consider maps

$$
\begin{aligned}
& H^{2 *, *}(X ; \mathbb{Z} / p) \stackrel{i_{!}}{\longrightarrow} H^{2 p *, p *}\left(X^{p} \times_{S_{p}} E S_{p}\right) \\
& \stackrel{\Delta^{*}}{\longrightarrow} H^{*, *}\left(X \times B S_{p} ; \mathbb{Z} / p\right) \cong H^{*, *}(X ; \mathbb{Z} / p) \otimes_{H^{*, *}(p t ; \mathbb{Z} / p)} H^{*, *}\left(B S_{p} ; \mathbb{Z} / p\right),
\end{aligned}
$$

where $i_{\text {! }}$ is the Gysin map for the $p$-th external power, and $\Delta$ is the diagonal map. For $\operatorname{deg}(x)=(2 n, n)$, the reduced powers are defined as

$$
\Delta^{*} i_{!}(x)=\sum P^{i}(x) \otimes Y^{n-i}+\beta P^{i}(x) \otimes W Y^{n-i-1} .
$$

Hence $\operatorname{deg}\left(P^{i}\right)=\operatorname{deg}\left(Y^{i}\right)=\operatorname{deg}\left(y^{i(p-1)}\right)=(2 i(p-1), i(p-1))$. Voevodsky defined

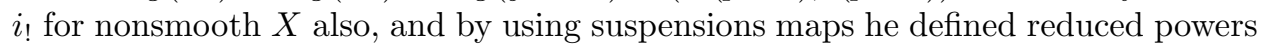
for all degree elements in $H^{*, *}(X ; \mathbb{Z} / p)$ for all $X$ [Vo3.

Moreover, we can see (Hu-Krríž [HK]) that

$$
H^{*, *}\left(B G L_{n} ; \mathbb{Z} / p\right) \cong \mathbb{Z} / p\left[c_{1}, \ldots, c_{n}\right] \otimes H^{*, *}(p t ; \mathbb{Z} / p),
$$

where the Chern class $c_{i}$ with $\operatorname{deg}\left(c_{i}\right)=(2 i, i)$ is identified with the elementary symmetric polynomial in $H^{*, *}\left(\left(\mathbb{P}^{\infty}\right)^{n} ; \mathbb{Z} / p\right)$. So we can define the Chern class $\rho^{*}\left(c_{i}\right) \in H^{2 *, *}(B G ; \mathbb{Z} / p)$ for each representation $\rho: G \rightarrow G L_{n}$.

\section{4. $H^{*, *}(X ; \mathbb{Z} / p) / \operatorname{Ker}\left(t_{\mathbb{C}}\right)$ AND THE OpERATION $Q_{i}$}

In this section we assume that $X$ is smooth and $k=\mathbb{C}$. Even in this case the motivic cohomology $H^{*, *}(X ; \mathbb{Z} / p)$ seems difficult, in general. Hence we consider a bigraded ring which is computable only by using the algebraic topology of $H^{*}(X(\mathbb{C}) ; \mathbb{Z} / p)$. Define a bidegree algebra by

$$
h^{*, *}(X ; \mathbb{Z} / p)=\bigoplus_{m, n} H^{m, n}(X ; \mathbb{Z} / p) / \operatorname{Ker}\left(t_{\mathbb{C}}^{m, n}\right) .
$$

Since $t_{\mathbb{C}}^{*, *}(\tau)=1$, it is almost immediate that there is the injection of bidegree $\mathbb{Z} / p[\tau]$-algebras

$$
h^{*, *}(X ; \mathbb{Z} / p) \hookrightarrow H^{*}(X(\mathbb{C}) ; \mathbb{Z} / p) \otimes \mathbb{Z} / p\left[\tau, \tau^{-1}\right],
$$

where the bidegree of $x \in H^{n}(X(\mathbb{C}) ; \mathbb{Z} / p)$ is $(n, n)$. (This also holds when $k \subset \mathbb{C}$ and $k$ has a primitive $p$-th root of 1 .)

Suppose the $B(n, p)$ condition holds. By the isomorphisms $(B, p),(\mathrm{L}-\mathrm{E}),(\mathrm{E} 1)$ and (E2), we have

$$
H^{n, n}(X ; \mathbb{Z} / p) \cong H_{L}^{n, n}(X ; \mathbb{Z} / p) \cong H_{e t}^{n}\left(X ; \mu_{p}^{\otimes n}\right) \cong H_{e t}^{n}(X ; \mathbb{Z} / p) \cong H^{n}(X(\mathbb{C}) ; \mathbb{Z} / p) .
$$

Hence we get the injection of bidegree $\mathbb{Z} / p[\tau]$-algebras

$$
H^{*}(X(\mathbb{C}) ; \mathbb{Z} / p) \otimes \mathbb{Z} / p[\tau] \hookrightarrow h^{* * *}(X ; \mathbb{Z} / p)
$$

Thus there exist a $\mathbb{Z} / p$-basis $\left\{a_{I}\right\}$ of $H^{*}(X(\mathbb{C}) ; \mathbb{Z} / p)$ and a $\left|\frac{1}{2} a_{I}\right| \geq t_{I} \geq 0$ such that

$$
h^{* * *}(X ; \mathbb{Z} / p) \cong \bigoplus_{I} \mathbb{Z} / p[\tau]\left\{\tau^{-t_{I}} a_{I}\right\}
$$


Remark. Let $F_{i}=\operatorname{Im}\left(\bigoplus_{m} t_{\mathbb{C}}^{m, i}\right)$. When the $B(n, p)$ condition is satisfied, we have $\bigcup_{i} F_{i}=H^{*}(X(\mathbb{C}) ; \mathbb{Z} / p)$. We also have the interesting bigraded ring

$$
g r H^{*}(X(\mathbb{C}) ; \mathbb{Z} / p)=\bigoplus F_{i+1} / F_{i} \cong h^{*, *}(X ; \mathbb{Z} / p) /(\operatorname{Im} \tau)
$$

so that $\mathbb{Z} / p[\tau] \otimes g r H^{*}(X(\mathbb{C}) ; \mathbb{Z} / p)$ is additively isomorphic to $h^{* * *}(X ; \mathbb{Z} / p)$, while the ring structures are different.

Here we recall the Milnor primitive operations $Q_{0}=\beta$ and $Q_{i}=\left[Q_{i-1}, P^{p^{i-1}}\right]$ :

$$
Q_{i}: H^{*, *}(X ; \mathbb{Z} / p) \rightarrow H^{*+2 p^{i}-1, *+p^{i}-1}(X ; \mathbb{Z} / p),
$$

which is derivative, $Q_{i}(x y)=Q_{i}(x) y+x Q_{i}(y)$. Note also that $Q_{i}(\tau)=0$, because of the dimension of $H^{*, *}(p t ; \mathbb{Z} / p) \cong \mathbb{Z} / p[\tau]$.

Lemma 4.1. If $0 \neq Q_{i_{1}} \ldots Q_{i_{s}} x \in H^{2 *, *}(X ; \mathbb{Z} / p)$, then $x$ is a $\mathbb{Z} / p[\tau]$-module generator.

Proof. If $x=x^{\prime} \tau$, then $\tau Q_{i_{1}} \ldots Q_{i_{s}}\left(x^{\prime}\right) \neq 0$. But

$$
Q_{i_{1}} \ldots Q_{i_{s}}\left(x^{\prime}\right)=0 \in H^{2 *, *-1}(X ; \mathbb{Z} / p),
$$

since $H^{m, n}(X ; \mathbb{Z} / p)=0$ for $m>2 n$.

Define the weight by $w(x)=2 n-m$ for an element $x \in H^{m, n}(X ; \mathbb{Z} / p)$, so that $w\left(x^{\prime}\right)=0$ for $x^{\prime} \in C H^{*}(X) / p$. Of course we get $w(x y)=w(x)+w(y)$, $w\left(P^{i} x\right)=w(x)$ and $w\left(Q_{i}(x)\right)=w(x)-1$.

Corollary 4.2. Suppose that $B(n, p)$ holds. If $x \in H^{n}(X(\mathbb{C}) ; \mathbb{Z} / p)$ and $Q_{i_{1}} \ldots Q_{i_{n}}(x)$ $\neq 0$, then there is a $\mathbb{Z} / p[\tau]$-module generator $x^{\prime} \in H^{n, n}(X ; \mathbb{Z} / p)$ so that $t_{\mathbb{C}}\left(x^{\prime}\right)=x$ and, for each $0 \leq k \leq n, Q_{i_{1}} \ldots Q_{i_{k}}\left(x^{\prime}\right)$ is also a $\mathbb{Z} / p[\tau]$-module generator of $H^{*, *}(X ; \mathbb{Z} / p)$.

Proof. By the $B(n, p)$ condition, $t_{\mathbb{C}}^{n, n}: H^{n, n}(X ; \mathbb{Z} / p) \cong H^{n}(X(\mathbb{C}) ; \mathbb{Z} / p)$. Hence there is an element $x^{\prime} \in H^{n, n}(X ; \mathbb{Z} / p)$ with $t_{\mathbb{C}}\left(x^{\prime}\right)=x$. This means $w\left(x^{\prime}\right)=n$ and $w\left(Q_{i_{1}} \ldots Q_{i_{n}}(x)\right)=0$. From the above lemma, we get the corollary.

Lemma 4.3. Suppose that $B(n, p)$ holds. If there is an $s>0$ with $p^{s} H^{n+1}(X(\mathbb{C}))_{(p)}$ $\subset t_{\mathbb{C}}\left(H^{n+1, n}(X)_{(p)}\right)$, then

$$
\operatorname{Im}\left(H^{n+1}(X(\mathbb{C})) \rightarrow H^{n+1}(X(\mathbb{C}) ; \mathbb{Z} / p)\right)=\operatorname{Im}\left(\left(H^{n+1, n}(X) \rightarrow H^{n+1}(X(\mathbb{C}) ; \mathbb{Z} / p)\right) .\right.
$$

Proof. Consider the following diagram:

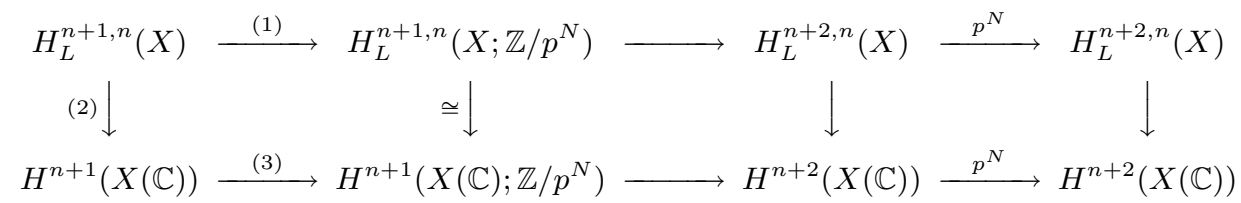

where $H^{*}(-)$ means $H^{*}(-; \mathbb{Z})_{(p)}$ and the rows are exact.

Let $H^{n+i}(X(\mathbb{C})) \cong F_{i} \oplus T_{i}$ and $H_{L}^{n+i, n}(X) \cong F_{i}^{\prime} \oplus T_{i}^{\prime} \oplus D_{i}$, where $F_{i}, F_{i}^{\prime}$ are free, $T_{i}, T_{i}^{\prime}$ are non- $p$-divisible torsion and $D_{i}$ are $p$-divisible submodules. Take $N$ and $s$ so that $p^{N}>p^{s}>\left|T_{i}\right|,\left|T_{i}^{\prime}\right|$ for $i=1,2$. Hence $H_{L}^{n+1, n}\left(X ; \mathbb{Z} / p^{N}\right) \cong$ $H^{n+1}\left(X(\mathbb{C}) ; \mathbb{Z} / p^{N}\right) \cong F_{1} / p^{N} \oplus T_{1} \oplus T_{2}$.

By the $B(n, p)$ condition, $H^{n+1, n}(X) \cong H_{L}^{n+1, n}(X)$, and the map (2) is identified with the realization map. So $p^{s}\left(F_{1} \oplus T_{1}\right)=p^{s} F_{1} \subset$ Image(2). Therefore there is the quotient map $F_{1} / p^{s} \oplus T_{1} \oplus T_{2} \rightarrow \operatorname{Coker}(1)$. On the other hand, 
$\operatorname{Ker}\left(p^{N}\right) \mid H_{L}^{n+2, n}(X) \cong\left(\operatorname{Ker}\left(p^{N}\right) \mid D_{2}\right) \oplus T_{2}^{\prime} \cong\left(\mathbb{Z} / p^{N}\right)^{k} \oplus T_{2}^{\prime}$. Hence if $k \neq 0$, then it is a contradiction to $\operatorname{Ker}\left(p^{N}\right)=\operatorname{Coker}(1)$. Hence we get $\operatorname{Coker}(1) \cong T_{2}^{\prime}$ and hence $\operatorname{Im}(3)(2)=F_{1} / p^{N} \oplus T_{1}$.

Corollary 4.4. Suppose that $B(n, p)$ holds and $t_{\mathbb{C}}^{n+1, n} \otimes \mathbb{Q}: H^{n+1, n}(X) \otimes \mathbb{Q} \rightarrow$ $H^{n+1}(X(\mathbb{C})) \otimes \mathbb{Q}$ is epic. If $x \in \operatorname{Im}\left(H^{n+1}(X(\mathbb{C})) \rightarrow H^{n+1}(X(\mathbb{C}) ; \mathbb{Z} / p)\right)$ and $Q_{i_{1} \ldots Q_{i_{n-1}}}(x) \neq 0$, then there is an element $x^{\prime} \in H^{n+1, n}(X)_{(p)}$ so that $t_{\mathbb{C}}\left(x^{\prime}\right)=x$ and, for each $0 \leq k \leq n-1, Q_{i_{1}} \ldots Q_{i_{k}}(x)$ is also a $\mathbb{Z} / p[\tau]$-module generator of $H^{*, *}(X ; \mathbb{Z} / p)$.

Here we mention the case $n=1$. Totaro showed $\left[\right.$ To2 that $C H^{*}(B G) \otimes \mathbb{Q} \cong$ $H^{*}(B G) \otimes \mathbb{Q}$ for any complex algebraic group $G$. Hence $C H^{1}(B G) \rightarrow H^{2}(B G)$ is epic; indeed, he also showed that this map is an isomorphism. As for $K 3$ surfaces, $C H^{*}(X) \otimes \mathbb{Q} \rightarrow H^{*}(X(\mathbb{C})) \otimes \mathbb{Q}$ is not epic and $H_{L}^{3,1}(X)$ contains $p$-divisible elements.

Now we consider some examples. The mod 2 cohomology of $B O(n)$ is $H^{*}(B O(n) ; \mathbb{Z} / 2) \cong \mathbb{Z} / 2\left[w_{1}, \ldots, w_{n}\right]$, where the Stiefel-Whitney class $w_{i}$ restricts the elementary symmetric polynomial in $H^{*}\left(B(\mathbb{Z} / 2)^{n} ; \mathbb{Z} / 2\right) \cong \mathbb{Z} / 2\left[x_{1}, \ldots, x_{n}\right]$. Each element $w_{i}^{2}$ is represented by the Chern class $c_{i}$ of the induced representation $O(n) \subset U(n)$. Hence $c_{i} \in C H^{*}(B O(n) ; \mathbb{Z} / 2)=H^{2 * * *}(B O(n) ; \mathbb{Z} / 2)$.

Proposition 4.5. $h^{* * *}(B O(n) ; \mathbb{Z} / 2) \supset \mathbb{Z} / 2\left[c_{1}, \ldots, c_{n}\right] \otimes \Delta\left(w_{1}, \ldots, w_{n}\right) \otimes \mathbb{Z} / 2[\tau]$, where $\operatorname{deg}\left(c_{i}\right)=(2 i, i)$, $\operatorname{deg}\left(w_{i}\right)=(i, i)$ and $w_{i}^{2}=\tau^{i} c_{i}$.

Since $Q_{i-1} \ldots Q_{0}\left(w_{i}\right) \neq 0$, each $w_{i}$ is a $\mathbb{Z} / 2[\tau]$-module generator. However, even $h^{*, *}(B O(n) ; \mathbb{Z} / 2)$ seems very complicated. Consider the case $X=B O(3)$. The cohomology operations act by

$$
\begin{gathered}
w_{2} \stackrel{S q^{1}}{\longrightarrow} w_{1} w_{2}+w_{3} \stackrel{S q^{2}}{\longrightarrow} w_{2} w_{1}^{3}+w_{1}^{2} w_{3}+w_{1} w_{2}^{2}+w_{2} w_{3} \stackrel{S q^{1}}{\longrightarrow} w_{1}^{2} w_{2}^{2}+w_{3}^{2}, \\
w_{3} \stackrel{S q^{1}}{\longrightarrow} w_{3} w_{1} \stackrel{S q^{2}}{\longrightarrow} \quad w_{1} w_{2} w_{3} .
\end{gathered}
$$

Theorem 4.6. There is the isomorphism

$h^{*, *}(B O(3) ; \mathbb{Z} / 2) \cong \mathbb{Z} / 2\left[c_{1}, c_{2}, c_{3}\right]\left\{1, w_{1}, w_{2}, Q_{0} w_{2}, Q_{1} w_{2}, w_{3}, Q_{0} w_{3}, Q_{1} w_{3}\right\} \otimes \mathbb{Z} / 2[\tau]$. where $Q_{0} w_{2}=\tau^{-1}\left(w_{1} w_{2}+w_{3}\right), \ldots, Q_{1} w_{3}=\tau^{-2} w_{1} w_{2} w_{3}$.

W. S. Wilson ([RWY], $[\mathrm{KY}])$ found a good $Q(i)=\Lambda\left(Q_{0}, \ldots, Q_{i}\right)$-module decomposition for $X=B O(n)$, namely,

$$
H^{*}(X ; \mathbb{Z} / 2)=\bigoplus_{i=-1}^{\infty} Q(i) G_{i} \quad \text { with } \quad Q_{0} \ldots Q_{i} G_{i} \in t_{\mathbb{C}}\left(C H^{*}(X)\right)
$$

Here $G_{k-1}$ is quite complicated; namely, it is generated by symmetric functions

$$
\Sigma x_{1}^{2 i_{1}+1} \ldots x_{k}^{2 i_{k}+1} x_{k+1}^{2 j_{1}} \ldots x_{k+q}^{2 j_{q}}, \quad k+q \leq n
$$

with $0 \leq i_{1} \leq \ldots \leq i_{k}$ and $0 \leq j_{1} \leq \ldots \leq j_{q}$; and if the number of $j$ equal to $j_{u}$ is odd, then there is some $s \leq k$ such that $2 i_{s}+2^{s}<2 j_{u}<2 i_{s}+2^{s+1}$.

Then $w\left(G_{i}\right) \geq i+1$ in $h^{*, *}(X ; \mathbb{Z} / p)$, and so we have

Proposition 4.7. Letting $w\left(G_{i}\right)=i+1$, we have the monomorphism

$$
h^{*, *}(B O(n) ; \mathbb{Z} / 2) \subset\left(\bigoplus_{i} Q(i) G_{i}\right) \otimes \mathbb{Z} / 2[\tau] .
$$


One interesting problem is whether the above injection is really an isomorphism. The similar decomposition holds for $X=(B \mathbb{Z} / p)^{n}$, and the above injection is an isomorphism. (See Lemma 5.6 below.) The case $X=B O(3)$ is also an isomorphism. Since the direct decomposition of $B O(3) \cong B S O(3) \times B \mathbb{Z} / 2$ is complicated, we only write here that of $S O(3)$ :

$$
\begin{gathered}
H^{*}(B S O(3) ; \mathbb{Z} / 2) \cong \mathbb{Z} / 2\left[w_{2}, w_{3}\right] \cong \mathbb{Z} / 2\left[c_{2}, c_{3}\right]\left\{1, w_{2}, w_{3}=Q_{0} w_{2}, w_{2} w_{3}=Q_{1} w_{2}\right\} \\
\cong \mathbb{Z} / 2\left[c_{2}, c_{3}\right]\left\{w_{2}, Q_{0} w_{2}, Q_{1} w_{2}, c_{3}=Q_{0} Q_{1} w_{2}\right\} \oplus \mathbb{Z} / 2\left[c_{2}\right] \\
\cong \mathbb{Z} / 2\left[c_{2}, c_{3}\right] Q(1)\left\{w_{2}\right\} \oplus \mathbb{Z} / 2\left[c_{2}\right] .
\end{gathered}
$$

Since there is the isomorphism $O(2 n+1) \cong S O(2 n+1) \times \mathbb{Z} / 2$, the cohomology of $B S O(2 n+1)$ is reduced from that of $B O(2 n+1)$. However, the situation for $B O(2 n)$ is different. In the next section, we will study $B S O(4)$ for details.

The extraspecial 2-group $2_{+}^{1+2 n}$ is the $n$-th central product of the dihedral group $D_{8}$ of order 8 . It has a central extension

$$
0 \rightarrow \mathbb{Z} / 2 \rightarrow G \rightarrow V=\bigoplus^{2 n} \mathbb{Z} / 2 \rightarrow 0
$$

Let $H^{*}(B V ; \mathbb{Z} / 2) \cong \mathbb{Z} / 2\left[x_{1}, \ldots, x_{2 n}\right]$. Then Quillen proved [Q]

$$
H^{*}(B G ; \mathbb{Z} / 2) \cong \mathbb{Z} / 2\left[x_{1}, \ldots, x_{2 n}\right] /\left(f, Q_{0} f, \ldots, Q_{n-2} f\right) \otimes \mathbb{Z} / 2\left[w_{2^{n}}\right] .
$$

Here $w_{2^{n}}$ is the Stiefel-Whitney class of the real $2^{n}$-dimensional irreducible representation which restricts nonzero on the center, and $f=\sum_{i} x_{2 i-1} x_{2 i} \in H^{2}(B V ; \mathbb{Z} / 2)$ represents the central extension (4.4).

Letting $y_{i}=x_{i}^{2}$ in $H^{*}(B G ; \mathbb{Z} / 2)$, we can write $f^{2}=\sum y_{2 i-1} y_{2 i}$ and

$$
\begin{gathered}
\left(Q_{k-1} f\right)^{2}=Q_{0} Q_{k} f=\sum y_{2 i-1}^{2^{k}} y_{2 i}-y_{2 i-1} y_{2 i}^{2^{k}}, \\
Q_{k-1} f=\sum y_{2 i-1}^{2^{k-1}} x_{2 i}-x_{2 i-1} y_{2 i}^{2^{k-1}} .
\end{gathered}
$$

Now we consider the motivic cohomology $H^{*, *}(B G ; \mathbb{Z} / 2)$ and change $y_{i}=\tau^{-1} x_{i}^{2}$. Since $f=0 \in H^{2,2}(B G ; \mathbb{Z} / 2)$, we can see that $Q_{k-1} f=0$ and $Q_{k} Q_{0}(f)=0$ also in $H^{*, *}(B G ; \mathbb{Z} / 2)$. However, for general $n, \sum y_{2 i} y_{2 i-1} \neq 0$ in $H^{*, *}(B G ; \mathbb{Z} / 2)$. Let

$$
\begin{aligned}
A= & \left(\mathbb{Z} / 2\left[y_{1}, \ldots, y_{2 n}, c_{2^{n}}\right] /\left(Q_{0} Q_{k} f, \ldots, Q_{0} Q_{n} f\right)\right. \\
& \left.\otimes \Delta\left(x_{1}, \ldots, x_{2 n}, w_{2^{n}}\right) /\left(f, Q_{0} f, \ldots, Q_{n-2} f\right)\right) \otimes \mathbb{Z} / 2[\tau] .
\end{aligned}
$$

Lemma 4.8. For $G=2_{+}^{1+2 n}$, there is a map $A \rightarrow H^{* * *}(B G ; \mathbb{Z} / 2)$ which induces the injection $A /\left(f^{2}\right) \subset h^{* * *}(B G ; \mathbb{Z} / 2)$.

When $m=0,1,-1 \bmod 8$ and $m>0$, we say that $\operatorname{Spin}(m)$ is real type $\mathbf{Q}$. When $\operatorname{Spin}(m)$ is real type, from Quillen, we know that $H^{*}(\operatorname{BSpin}(m) ; \mathbb{Z} / 2) \subset$ $H^{*}(B G ; \mathbb{Z} / 2)$, where $G=2_{+}^{2 h+1}$ and $h$ is the Hurwitz number (for details see $\mathrm{Q}$ ).

Corollary 4.9. Let $G=\operatorname{Spin}(m)$ be real type with Hurwitz number $h$, and let

$$
\begin{aligned}
A= & \left(\mathbb{Z} / 2\left[c_{2}, c_{3}, \ldots, c_{m}, c_{2^{h}}\right] /\left(\left(Q_{1} Q_{0} w_{2}\right), \ldots,\left(Q_{h} Q_{0} w_{2}\right)\right)\right. \\
& \left.\otimes \Delta\left(w_{2}, \ldots, w_{m}, w_{2^{h}}\right) /\left(w_{2}, Q_{0} w_{2}, \ldots, Q_{h-2} w_{2}\right)\right) \otimes \mathbb{Z} / 2[\tau],
\end{aligned}
$$

where $w_{i}, i \leq m$ (resp. $w_{2^{h}}$ ) is the Stiefel-Whitney class of the usual $S O(m)$ representation (resp. of the irreducible $2^{h}$-dimensional spin representation). Then we have a map $A \rightarrow H^{*, *}(B G ; \mathbb{Z} / 2)$ which induces the injection

$$
A /\left(c_{2}\right) \subset h^{* * *}(B G ; \mathbb{Z} / 2) .
$$


We study $\operatorname{Spin}(7)$ and the exceptional Lie group $G_{2}$. The cohomology of $G_{2}$ is given by $H^{*}\left(B G_{2} ; \mathbb{Z} / 2\right) \cong \mathbb{Z} / 2\left[w_{4}, w_{6}, w_{7}\right]$, where $w_{i}$ is the Stiefel-Whitney class of the inclusion $G_{2} \subset S O(7)$. The cohomology $H^{*}(B \operatorname{Spin}(7) ; \mathbb{Z} / 2) \cong H^{*}\left(B G_{2} ; \mathbb{Z} / 2\right) \otimes$ $\mathbb{Z} / 2\left[w_{8}\right]$.

Corollary 4.10. Let $A=\mathbb{Z} / 2\left[c_{2}, c_{4}, c_{6}, c_{7}\right] \otimes \Delta\left(w_{4}, w_{6}, w_{7}\right) \otimes \mathbb{Z} / 2[\tau]$. Then there is the map $A \rightarrow H^{*, *}\left(B G_{2} ; \mathbb{Z} / 2\right)$ which induces the injection $A /\left(c_{2}\right) \subset h^{*, *}\left(B G_{2} ; \mathbb{Z} / 2\right)$.

Remark. Similar facts hold for BSpin(7) tensoring $\mathbb{Z} / 2\left[c_{8}\right]$.

The cohomology operations are given by

$$
\begin{gathered}
w_{4} \stackrel{S q^{2}}{\longrightarrow} w_{6} \stackrel{S q^{1}}{\longrightarrow} w_{7} \stackrel{S q^{4}}{\longrightarrow} w_{4} w_{7} \stackrel{S q^{2}}{\longrightarrow} w_{7} w_{6} \stackrel{S q^{1}}{\longrightarrow} w_{7}^{2}, \\
Q_{1} Q_{0}\left(w_{4} w_{6}\right)=w_{7}^{2}, \quad Q_{2} Q_{1} Q_{0}\left(w_{4} w_{6} w_{7}\right)=w_{7}^{4} .
\end{gathered}
$$

Proposition 4.11. Let $w\left(w_{4}\right)=2, w\left(w_{(4,6)}\right)=2$ and $w\left(w_{(4,6,7)}\right)=3$ with $t_{\mathbb{C}}\left(w_{\left(i_{1}, \ldots, i_{n}\right)}\right)=w_{i_{1}} \ldots w_{i_{n}}$. Then $h^{*, *}\left(B G_{2} ; \mathbb{Z} / 2\right)$ is a subalgebra of $\mathbb{Z} / p[\tau] \otimes \mathbb{Z} / 2\left[c_{4}, c_{6}, c_{7}\right] \otimes \mathbb{Z} / 2\left\{1, w_{4}, S q^{2} w_{4}, Q_{1} w_{4}, Q_{2} w_{4}, S q^{2} Q_{2} w_{4}, w_{(4,6)}, w_{(4,6,7)}\right\}$.

Remark. If $t_{\mathbb{C}}^{4,3} \otimes \mathbb{Q}$ is epic, then we can take $w_{4} \in h^{4,3}(B G ; \mathbb{Z} / 2)$, i.e., $w\left(w_{4}\right)=2$.

The kernel $\operatorname{Ker}\left(t_{\mathbb{C}}\right)^{2 *, *}$ is not so big for $X=B G_{2}$. Indeed, it is known [Y3] that $C H^{*}\left(B G_{2}\right) / 2 \cong \mathbb{Z} / 2\left[c_{2}, c_{4}, c_{6}, c_{7}\right] /\left(r c_{2}^{2}, c_{2} c_{7}\right), \quad$ where $r=0$ or 1.

The cohomology operations are given in $H^{*}(B S O(7) ; \mathbb{Z} / 2)$ by

$$
Q_{1} Q_{0} w_{2}=w_{3}^{2}, \quad Q_{2} Q_{0} w_{2}=w_{5}^{2}, \quad Q_{3} Q_{0} w_{2}=w_{7}^{2} w_{2}^{2}+w_{6}^{2} w_{3}^{2}+w_{5}^{2} w_{4}^{2} .
$$

Hence we have $c_{3}=0, c_{5}=0$ and $c_{2} c_{7}=0$ in $C H^{*}\left(B G_{2}\right) / 2$, but $c_{2} \neq 0$.

From here we consider the case $p=o d d$. One of the easiest examples is the case $G=P G L_{3}$ and $p=3$. The mod 3 cohomology is given by ([KY], [Ve1]

$$
\left(\mathbb{Z} / 3\left[y_{2}\right]\left\{y^{2}\right\} \oplus \mathbb{Z} / 3\left\{1, y_{2}, y_{3}, y_{7}\right\}\left[y_{8}\right]\right) \otimes \mathbb{Z} / 3\left[y_{12}\right]
$$

It is known that $y_{2}^{2}, y_{2}^{3}, y_{8}^{2}$ and $y_{12}$ are represented by Chern classes. Moreover, $Q_{1} Q_{0}\left(y_{2}\right)=y_{8}$. Hence these elements are in the Chow ring; namely,

$$
h^{2 *, *}\left(B P G L_{3} ; \mathbb{Z} / 3\right) \cong\left(\mathbb{Z} / 3\left[y_{2}\right]\left\{y_{2}^{2}\right\} \oplus \mathbb{Z} / 3\left[y_{8}\right]\right) \otimes \mathbb{Z} / 3\left[y_{12}\right] .
$$

The cohomology operations are given by

$$
y_{2} \stackrel{\beta}{\longrightarrow} y_{3} \stackrel{P^{1}}{\longrightarrow} y_{7} \stackrel{\beta}{\longrightarrow} y_{8} .
$$

Thus we get $h^{*, *}\left(P G L_{3} ; \mathbb{Z} / 3\right)$ completely.

Theorem 4.12. Letting $w\left(y_{2}\right)=2$, we have the isomorphism

$$
h^{*, *}\left(B P G L_{3} ; \mathbb{Z} / 3\right) \cong\left(\mathbb{Z} / 3\left[y_{2}\right]\left\{y^{2}\right\} \oplus \mathbb{Z} / 3\{1\} \oplus \mathbb{Z} / 3\left[y_{8}\right] \otimes Q(1)\left\{y_{2}\right\}\right) \otimes \mathbb{Z} / 3\left[y_{12}, \tau\right] .
$$

Next consider the extraspecial $p$-group $G=p_{+}^{1+2 n}$. When $n>2$, even the cohomology rings $H^{*}(B G ; \mathbb{Z} / p)$ are unknown, while it contains the subring [TeY1]

$$
R=\mathbb{Z} / p\left[y_{1}, \ldots, y_{2 n}, c_{p^{n}}\right] /\left(Q_{1} Q_{0} f, \ldots, Q_{n} Q_{0} f\right),
$$

where $f=\sum^{n} x_{2 i-1} x_{2 i}$ for $\beta x_{i}=y_{i}$ and $Q_{k} Q_{0} f=\sum y_{2 i-1} y_{2 i}^{p^{k}}-y_{2 i-1}^{p^{k}} y_{2 i}$. Since $f=0 \in H^{2,2}(B G ; \mathbb{Z} / p)$, we have

Proposition 4.13. There is the injection

$$
R \otimes \mathbb{Z} / p[\tau] \hookrightarrow H^{*, *}\left(B p_{+}^{1+2 n} ; \mathbb{Z} / p\right) .
$$


We consider here other arguments for a different but similar group. Let $\tilde{p}_{+}^{1+2 n}$ be the central product of $p_{+}^{1+2 n}$ and the circle, i.e. $\tilde{p}_{+}^{1+2 n}=p_{+}^{1+2 n} \times_{C} S^{1}$, identifying $C \cong \mathbb{Z} / p \subset S^{1}$, where $C$ is the center. Let us write

$$
e_{A}=\prod_{0 \neq\left(\lambda_{1}, \lambda_{3}, \ldots, \lambda_{2 n-1}\right)}\left(\lambda_{1} y_{1}+\cdots+\lambda_{2 n-1} y_{2 n-1}\right) .
$$

If we localize by inverting $e_{A}$, then the cohomology of $\tilde{p}_{+}^{1+2 n}$ is expressed easily [Y2] as

$$
\left[e_{A}^{-1}\right] H^{*}\left(B \tilde{p}_{+}^{1+2 n} ; \mathbb{Z} / p\right) \cong\left[e_{A}^{-1}\right] R \otimes \Lambda\left(x_{1}, x_{3}, \ldots, x_{2 n-1}\right), \quad \beta\left(x_{i}\right)=y_{i} .
$$

Theorem 4.14. Letting $w\left(x_{i}\right)=1$, we have the ring isomorphism

$$
\left[e_{A}^{-1}\right] h^{*, *}\left(B\left(\tilde{p}_{+}^{1+2 n}\right) ; \mathbb{Z} / p\right) \cong\left[e_{A}^{-1}\right] R \otimes \mathbb{Z} / p[\tau] \otimes \Lambda\left(x_{1}, x_{3}, \ldots, x_{2 n-1}\right) .
$$

Proof. There is the splitting abelian subgroup $(\mathbb{Z} / p)^{n} \cong A \subset \tilde{p}_{+}^{1+2 n}$ such that

$$
h^{*, *}(B A ; \mathbb{Z} / p) \cong \mathbb{Z} / p\left[\tau, y_{1}, y_{3}, \ldots, y_{2 n-1}\right] \otimes \Lambda\left(x_{1}, x_{3}, \ldots, x_{2 n-1}\right) .
$$

Each monomial $x_{i_{1}} \ldots x_{i_{s}}, 1 \leq i_{1}, \ldots, i_{s} \leq 2 n-1$, is a $\mathbb{Z} / p[\tau]$-module generator in the above cohomology, hence also in the cohomology of $B \tilde{p}_{+}^{1+2 n}$.

We consider the case $n=1$ here. Let us write $E=p_{+}^{1+2}$ for each odd prime $p$. The ordinary cohomology is known by Lewis [Ly], [TeY2]; namely,

$$
\begin{aligned}
& H^{\text {even }}(B E) / p \cong\left(\mathbb{Z} / p\left[y_{1}, y_{2}\right] /\left(y_{1}^{p} y_{2}-y_{1} y_{2}^{p}\right) \oplus \mathbb{Z} / p\left\{c_{2}, \ldots, c_{p-1}\right\}\right) \otimes \mathbb{Z} / p\left[c_{p}\right], \\
& H^{\text {odd }}(B E) \cong \mathbb{Z} / p\left[y_{1}, y_{2}, c_{p}\right]\left\{a_{1}, a_{2}\right\} /\left(y_{1} a_{2}-y_{2} a_{1}, y_{1}^{p} a_{2}-y_{2}^{p} a_{1}\right), \quad\left|a_{i}\right|=3 .
\end{aligned}
$$

It is also known that $Q_{1}\left(a_{i}\right)=y_{i} c_{p}$ and $\operatorname{order}\left(c_{p}\right)=p^{2}$.

The group $2_{+}^{1+2}$ is the dihedral group $D_{8}$ of order 8 . The integral cohomologies are

$$
H^{\text {even }}\left(B D_{8}\right) / 2 \cong \mathbb{Z} / 2\left[y_{1}, y_{2}, c_{2}\right] /\left(y_{1} y_{2}\right), \quad H^{\text {odd }}\left(B D_{8}\right) \cong H^{\text {even }}\left(B D_{8}\right) / 2\{e\}
$$

where $c_{2}=w_{2}^{2}, e=\left(x_{1}+x_{2}\right) w_{2}$ in $H^{*}\left(B D_{8} ; \mathbb{Z} / 2\right) \cong \mathbb{Z} / 2\left[x_{1}, x_{2}, w_{2}\right] /\left(x_{1} x_{2}\right)$ and $Q_{1} e=\left(y_{1}+y_{2}\right) c_{2}, \operatorname{order}\left(c_{2}\right)=4$.

Theorem 4.15. For all primes $p$, we have the isomorphisms

$$
h^{*, *}\left(B p_{+}^{1+2} ; \mathbb{Z} / p\right) \cong\left(\left\{1, \partial_{p}^{-1}\right\}\left(H^{*}\left(B p_{+}^{1+2}\right) / p\right)-\left\{\partial_{p}^{-1} 1\right\}\right) \otimes \mathbb{Z} / p[\tau],
$$

where $w\left(H^{\text {even }}\left(B p_{+}^{1+2}\right) / p\right)=0, w\left(H^{\text {odd }}\left(B p_{+}^{1+2}\right)\right)=1$ and $w\left(\partial_{p}^{-1}(x)\right)=w(x)+1$.

Proof. We will prove this only for odd primes, since the proof for $p=2$ is similar. Since all elements in $H^{\text {even }}(B E)$ are generated by Chern classes, we have the isomorphism $h^{2 *, *}(B G ; \mathbb{Z} / p) \cong H^{\text {even }}(B E) / p$. We know $H^{\text {odd }}(B E ; \mathbb{Z} / p)$ is generated as an $H^{\text {even }}(B E) / p$-module by two elements $a_{1}, a_{2}$ such that $Q_{1} a_{i}=y_{i} c_{p}$ TeY2].

The $\bmod p$ cohomology is written additively, $H^{*}(B E ; \mathbb{Z} / p) \cong\left\{1, \partial_{p}^{-1}\right\} H^{*}(B E) / p$. Here $\partial_{p}$ is the (higher) Bockstein operator. All elements in $H^{\text {odd }}(B E)$ are just $p$ torsion, and we can take $a_{i}^{\prime} \in H^{2}(B E ; \mathbb{Z} / p)$ such that $\beta\left(a_{i}^{\prime}\right)=a_{i}$. Thus we take $a_{i}^{\prime} \in H^{2,2}(B E ; \mathbb{Z} / p)$ so that $a_{i} \in H^{3,2}(B E ; \mathbb{Z} / p)$.

Next consider elements $x=\partial_{p}^{-1}(y), y \in H^{\text {even }}(B E) / p$. If $y \in\left(\operatorname{Ideal}\left(y_{1}, y_{2}\right)\right)$, then $\partial_{p}^{-1}(y)=\sum x_{i} b_{i}$ for $b_{i} \in H^{\text {even }}(B E) / p$, and hence we can take $w\left(\partial_{p}^{-1}(y)\right)=1$. For other elements $y=c_{i} c_{p}^{n}, 2 \leq i \leq p-1$, it is known $\mathrm{Ly}$ that $c_{i}=\operatorname{Cor}_{M}^{E}\left(u^{i}\right)$ 
with $0 \neq u \in H^{2}(B \mathbb{Z} / p ; \mathbb{Z} / p)$ for a maximal abelian subgroup $M \cong \mathbb{Z} / p \times \mathbb{Z} / p$. Hence $y \in H^{2 *, *}(B E ; \mathbb{Z} / p)$ is also $p$-torsion. Considering the exact sequence

$$
\rightarrow H^{2 *-1, *}\left(B E ; \mathbb{Z} / p^{N}\right) \rightarrow H^{2 *, *}(B E) \stackrel{p^{N}}{\rightarrow} H^{2 *, *}(B E) \rightarrow,
$$

we get $w\left(\partial_{p}^{-1}(y)\right)=1$. The element $y=c_{p}^{n}$ is $p^{2}$-torsion in $H^{*}(B E ; \mathbb{Z} / p)$. Note that $\operatorname{Cor}_{M}^{E}\left(u^{p n}\right)=p c_{p}^{n}+k$ with $k \in \operatorname{Ideal}\left(y_{1}, y_{2}\right)$. Thus $y \in H^{2 *, *}(B E ; \mathbb{Z} / p)$ is also $p^{2}$-torsion. Then we can take $w\left(\partial_{p}^{-1}(y)\right)=1$. This completes the proof.

We easily compute the following results.

Corollary 4.16. For each prime $p$, there is an isomorphism

$$
h^{*, *}\left(B p_{+}^{1+2} ; \mathbb{Z} / p\right) \cong \mathbb{Z} / p[\tau] \otimes\left(\mathbb{Z} / p\{1\} \oplus Q^{\prime}(0) G_{0}^{\prime} \oplus Q(0) G_{0} \oplus Q(1) G_{1}\right),
$$

where $Q^{\prime}(0)=\Lambda\left(\beta_{p^{2}}\right), \beta_{p^{2}}$ is the $p^{2}$-torsion Bockstein operator, and if $p=2$, then

$$
\left\{\begin{array}{l}
G_{0}^{\prime} \cong \mathbb{Z} / 2\left[c_{2}\right]\left\{x_{1} w_{2}\right\}, \quad \beta_{4}\left(x_{1} w_{2}\right)=c_{2} \\
G_{0} \cong \mathbb{Z} / 2\left[y_{1}\right]\left\{x_{1}\right\} \oplus \mathbb{Z} / 2\left[y_{2}\right]\left\{x_{2}\right\} \oplus \mathbb{Z} / 2\left[c_{2}\right]\left\{x_{1} c_{2}\right\} \\
G_{1} \cong \mathbb{Z} / 2\left[y_{1}, y_{2}, c_{2}\right] /\left(y_{1} y_{2}\right)\left\{w_{2}\right\}
\end{array}\right.
$$

and if $p$ is an odd prime, then

$$
\left\{\begin{array}{l}
G_{0}^{\prime} \cong \mathbb{Z} / p\left[c_{p}\right]\left\{c_{p}^{\prime}\right\}, \quad \beta_{p^{2}}\left(c_{p}^{\prime}\right)=c_{p}, \\
G_{0} \cong \mathbb{Z} / p\left[y_{1}, y_{2}\right]\left\{x_{1}, x_{2}\right\} /\left(y_{2} x_{1}-y_{1} x_{2}, y_{2}^{p} x_{1}-y_{1}^{p} x_{2}\right) \oplus \mathbb{Z} / p\left[c_{p}\right]\left\{c_{2}^{\prime}, \ldots, c_{p-1}^{\prime}\right\}, \\
G_{1} \cong \mathbb{Z} / p\left[y_{1}, y_{2}, c_{p}\right]\left\{a_{1}^{\prime}, a_{2}^{\prime}\right\} /\left(y_{2} a_{1}^{\prime}-y_{1} a_{2}^{\prime}, y_{2}^{p} a_{1}^{\prime}-y_{1}^{p} a_{2}^{\prime}\right), \quad \beta\left(a_{i}^{\prime}\right)=a_{i}, \beta\left(c_{i}^{\prime}\right)=c_{i} .
\end{array}\right.
$$

\section{5. $B P$-THEORY AND $\operatorname{Ker} t_{\mathbb{C}}^{2 *, *}$}

In this section, we always assume $k=\mathbb{C}$. Even this case it seems difficult to know $\operatorname{Ker} t_{\mathbb{C}}$. For Chow rings $C H^{*}(X)$, Totaro found a good way to get nonzero elements in Ker $t_{\mathbb{C}}$. Let $M U^{*}(-)$ (resp. $B P^{*}(-)$ ) be the complex cobordism theory (resp. Brown-Peterson theory) with the coefficient ring $M U^{*}=M U^{*}(p t)=$ $\mathbb{Z}\left[x_{1}, \ldots\right],\left|x_{i}\right|=-2 i\left(\operatorname{resp} . B P^{*}=\mathbb{Z}_{(p)}\left[v_{1}, \ldots\right],\left|v_{i}\right|=-2\left(p^{i}-1\right)\right)$. The Thom map induces $\rho: M U^{*}(X(\mathbb{C})) \otimes_{M U^{*}} \mathbb{Z} \rightarrow H^{*}(X(\mathbb{C}) ; \mathbb{Z})$. Totaro constructed To1 the map

$$
\tilde{c l}: C H^{*}(X) \rightarrow M U^{*}(X(\mathbb{C})) \otimes_{M U *} \mathbb{Z}
$$

such that the composition $\rho \tilde{c l}$ is the usual cycle map $c l=t_{\mathbb{C}}^{2 *, *}$, which is also the realization map.

In this section, hereafter, $X$ is just a topological space, e.g., $X(\mathbb{C})$, to simplify the notation. Since $B P^{*}(X) \cong B P^{*} \otimes_{M U_{(p)}^{*}} M U^{*}(X)_{(p)}$, the similar fact holds for $B P$ theory. Let $P(n)^{*}=B P^{*} /\left(p, v_{1}, \ldots, v_{n-1}\right)$, e.g., $P(0)^{*}=B P^{*}, P(1)^{*}=B P^{*} / p$ and $P(\infty)^{*}=\mathbb{Z} / p$. Then there are cohomology theories $P(n)^{*}(-)$ with the coefficient $P(n)^{*}(p t) \cong P(n)^{*}$, e.g., $P(0)^{*}(X)=B P^{*}(X), P(1)^{*}(X)=B P^{*}(X ; \mathbb{Z} / p)$ and $P(\infty)^{*}(X)=H^{*}(X ; \mathbb{Z} / p)$. Hence there are maps of cohomology theories

$$
\begin{aligned}
c l_{p}: C H^{*}(-) / p & \rightarrow B P^{*}(-) \otimes_{B P^{*}} \mathbb{Z} / p \rightarrow \ldots \rightarrow P(n)^{*}(-) \otimes_{P(n)^{*}} \mathbb{Z} / p \\
& \rightarrow P(n+1)^{*}(-) \otimes_{P(n+1)^{*}} \mathbb{Z} / p \rightarrow \ldots \rightarrow H^{*}(-; \mathbb{Z} / p)
\end{aligned}
$$

such that the composition is the cycle map $c l_{p}=t_{\mathbb{C}}$. The Morava $K$-theory is defined by $K(n)^{*}(X)=P(n)^{*}(X) \otimes_{P(n)^{*}} K(n)^{*}$, where $K(n)^{*}=\mathbb{Z} / p\left[v_{n}, v_{n}^{-1}\right]$. In 
general, $K(n)^{*}(X) \neq K(n)^{*} \otimes_{B P^{*}} B P^{*}(X)$. However, when $K(n)^{\text {odd }}(X)=0$, it is known [RW] that

$$
P(n)^{*}(X) \cong B P^{*}(X) \otimes_{B P^{*}} P(n)^{*}, \quad K(n)^{*}(X) \cong B P^{*}(X) \otimes_{B P^{*}} K(n)^{*} .
$$

We know that $K(n)^{\text {odd }}(B G)=0$ for many cases, while Kř́ž showed $K(n)^{*}\left(B G^{\prime}\right) \neq$ 0 for some fine group $G^{\prime}$.

One useful tool for computing $B P^{*}(X)$ is the Atiyah-Hirzebruch spectral sequence [TeY2], $\mathrm{KY}]$

$$
E_{2}^{*, *}=H^{*}(X) \otimes B P^{*} \Longrightarrow B P^{*}(X) .
$$

It is known that $d_{2 p^{i}-1}(x)=v_{i} \otimes Q_{i}(x) \bmod \left(M_{i}\right)$, where $M_{i}$ is the ideal of $E_{2 p^{i}-1}^{* * *}$ generated by elements in $\left(p, v_{1}, \ldots, v_{i-1}\right) E_{2}^{*, *}$. Here we assume that $H^{*}(X)$ has no higher $p$-torsion and that

(5.2) All nonzero differentials are of the form

$$
d_{2 p^{i}-1}(x)=v_{i} \otimes Q_{i}(x) \bmod \left(M_{i}\right) .
$$

Let us write

$$
\operatorname{gr} B P^{*}(X) \cong E_{\infty}^{*, *} \cong A \oplus B
$$

where $A$ (resp. $B$ ) is a $B P^{*}$-module generated by nonzero elements in $H^{*}(X) / p$ (resp. $p H^{*}(X) \oplus E_{\infty}^{*, \text { minus }}$ ), so that $B \subset \operatorname{Ker}\left(\rho_{p}\right)$. We can write

$$
A \cong \bigoplus_{n=-1}^{\infty} P(n+1)^{*} \tilde{G}_{n}
$$

by the prime invariant ideal theorem of Landweber; if $P(n)^{*} /(a)$ is a $B P^{*}(B P)$ module, then $a=v_{n}^{s}$ for some $s \geq 1$.

Take a nonzero element $\tilde{g}_{n} \in \tilde{G}_{n}$ for $n \geq 2$. Since $\tilde{g}_{n}$ is $\left(p, \ldots, v_{n}\right)$-torsion, there is $g_{(n, s)} \in E_{2 p^{s}-1}^{*, 0}$ such that $d_{2 p^{s}-1}\left(g_{(n, s)}\right)=v_{s} \otimes \tilde{g}_{n}$ for each $1 \leq s \leq n$. Let the $B P^{*}$ module in $E_{2 p^{s}-1}^{*, *}$ generated by $g_{(n, s)}$ be isomorphic to a $P\left(s^{\prime}+1\right)^{*}$-free module for $s^{\prime}<s$. Here note that if $s^{\prime} \neq s-1$, then $\operatorname{Ideal}\left(v_{s^{\prime}+1}, . ., v_{s-1}\right)\left\{g_{n, s}\right\} \subset \operatorname{Ker}\left(d_{2 p^{s}-1}\right)$. In any case, we can take $g_{(n, s, t)} \in H^{*}(X) / p$ for $t<s^{\prime}$ such that $d_{2 p^{t}-1}\left(g_{(n, s, t)}\right)=$ $v_{t} \otimes g_{(n, s)}$. Continuing this argument we can take

$$
\tilde{g}_{n} \stackrel{Q_{s_{1}}}{\longleftarrow} g_{\left(n, s_{1}\right)} \stackrel{Q_{s_{2}}}{\longleftarrow} g_{\left(n, s_{1}, s_{2}\right)} \longleftarrow \ldots \stackrel{Q_{s_{m}}}{\longleftarrow} g_{\left(n, s_{1}, \ldots, s_{m}\right)}
$$

for some $\left(n>s_{1}>\ldots>s_{m}\right)$.

Lemma 5.1. Let $H^{*}(X)_{(p)}$ have no higher p-torsion. Suppose (5.2) holds, and $A=\bigoplus_{n=-1} P(n+1)^{*} \tilde{G}_{n}$ in (5.3). Then there is the injection

$$
H^{*}(X ; \mathbb{Z} / p) \hookrightarrow \bigoplus_{n} Q(n) G_{n} \text { with } Q_{0} \ldots Q_{n} G_{n}=\tilde{G}_{n} .
$$

Proof. Let $H$ be a $\mathbb{Z} / p$-module generated by elements $g_{\left(n, s_{1}, \ldots, s_{m}\right)}$ discussed above. Define the map $j_{\mathbb{C}}: H \hookrightarrow \oplus Q(n) G_{n}$ by

$$
j_{\mathbb{C}}\left(g_{\left(n, s_{1}, \ldots, s_{m}\right)}\right)=Q_{s_{m}}^{-1} \ldots Q_{s_{1}}^{-1}\left(\tilde{g}_{n}\right)=Q_{0} \ldots \hat{Q}_{s_{m}} \ldots \hat{Q}_{s_{1}} \ldots Q_{n}\left(g_{n}\right), \quad Q_{0} \ldots Q_{n} g_{n}=\tilde{g}_{n} .
$$

Suppose $x \in H^{*}(X)_{(p)}-H$. Then by the assumption (5.3), $x$ is not a permanent cycle. Hence $d_{2 p^{i}-1}(x) \neq 0$ for some $i$, and so $Q_{i}(x) \neq 0$. Let $t$ be a largest number such that $Q_{i_{t}} \ldots Q_{i_{1}} Q_{i} x=\tilde{g} \neq 0$. Since $Q_{j}(\tilde{g})=0$ for all $j$, we know $\tilde{g}$ is a permanent cycle. This element $\tilde{g} \in E_{\infty}^{*, 0}$ generates a $P(N+1)^{*}$-module for $N=\max \left(i_{s}, \ldots, i_{1}, i\right)$. This means $x=\left(Q_{i}^{-1} Q_{i_{1}}^{-1} \ldots Q_{i_{s}}^{-1} \tilde{g}\right) \in H$. 
Let us write $Q(i, n)=\Lambda\left(Q_{i}, \ldots, Q_{n}\right)$, so that $Q(0, n)=Q(n)$.

Lemma 5.2. Let $H^{*}(X)_{(p)}$ have no higher p-torsion.

(1) If (5.2) is satisfied and, in (5.3),

$$
A=\bigoplus_{n=-1} P(n+1)^{*} \tilde{G}_{n} \quad \text { and } B \cong \bigoplus_{s=0} B P^{*}\left\{p, v_{1}, . ., v_{s}\right\} \tilde{K}_{s}
$$

then we have the isomorphisms

$$
\begin{gathered}
H^{*}(X) / p \cong\left(\tilde{G}_{-1} \oplus \tilde{G}_{0} \oplus \bigoplus_{n=1} Q(1, n) G_{n}^{\prime}-\bigoplus_{s=0}\left(Q(1, s) K_{s}^{\prime}-\tilde{K}_{s}^{\prime}\right)\right), \\
H^{*}(X ; \mathbb{Z} / p) \cong\left(\bigoplus_{n=-1} Q(n) G_{n}-\bigoplus_{s=0}\left(Q(s) K_{s}-\tilde{K}_{s}\right)\right)
\end{gathered}
$$

with $Q_{0} \ldots Q_{n} G_{n}=\tilde{G}_{n}, Q_{0} G_{n}=G_{n}^{\prime}$ and $Q_{0} \ldots Q_{s} K_{s}=\tilde{K}_{s}, Q_{0} K_{s}=K_{s}^{\prime}$.

(2) If $Q_{0} \ldots Q_{n} G_{n} \in \operatorname{Im}(\rho)$ and the degrees of $\tilde{K}_{s}$ and $\tilde{G}_{n}$ are even, then the converse also holds.

Proof. (1) Let $0 \neq x \in \tilde{K}_{s}$. Since $x$ is not a permanent cycle, $d_{2 p^{i}-1}(x) \neq 0$ and $Q_{i}(x) \neq 0$. Since $\left\{p, \ldots, v_{s}\right\} \tilde{K}_{s}$ are permanent cycles, we know $Q_{i}(x) \in E_{2 p^{i}-1}^{* *}$ is a $P(s+1)^{*}$-module, that is, $i=s+1$ by the Landweber invariant prime ideal theorem, and

$$
\bigoplus Q(n) G_{n} \supset Q(s) K_{s}
$$

Since $v_{i} x$ generates a free $B P^{*}$-module, $x \notin \operatorname{Im}\left(Q_{j}\right)$ for all $j$. Hence we get the injection

$$
H^{*}(X ; \mathbb{Z} / p) \hookrightarrow \bigoplus Q(n) G_{n}-\left(Q(s) K_{s}-\tilde{K}_{s}\right) .
$$

Let $x=Q_{i_{1}} \ldots Q_{i_{k}} g_{n}$ be in the right-hand side of the above injection, and such that $0 \neq Q_{i}(x) \in H^{*}(X ; \mathbb{Z} / p)$ but $x \notin H^{*}(X ; \mathbb{Z} / p)$. If $Q_{i}(x)$ is not a permanent cycle, then $v_{i} Q_{i}(x)$ is permanent, so $Q_{i}(x)$ must be in $\tilde{K}_{s}$ and hence $x \in Q(s) K_{s}$; this is a contradiction. Otherwise $Q_{i}(x)=\tilde{g}_{n}$ generates a $P(n)^{*}$-module and $Q_{i}(x)$ must be $\operatorname{Im}\left(Q_{j}\right)$ for all $j \leq n$. Hence $x \in H^{*}(X ; \mathbb{Z} / p)$.

(2) By induction on $i$, we assume $E_{2 p^{i-1}}^{*, *} \cong C(i) \oplus D(i)$, where

$$
\begin{gathered}
C(i)=P(i)^{*}\left(\bigoplus_{i \leq n} Q(i, n) Q_{i-1} \ldots Q_{0} G_{n}-\bigoplus_{i \leq s} Q(i, n) Q_{i-1} \ldots Q_{0} K_{s}\right) \oplus \bigoplus_{i-1 \leq s} B P^{*} \tilde{K}_{s}, \\
D(i)=\bigoplus_{n \leq i-1} P(n+1)^{*} \tilde{G}_{n} \oplus \bigoplus_{s \leq i-2} B P^{*}\left\{p, \ldots, v_{s}\right\} \tilde{K}_{s} .
\end{gathered}
$$

Here elements of $\tilde{K}_{s}$ and $D(i)$ are even dimensional. Hence all odd dimensional elements generate free $P(i)^{*}$-modules. Note that if $i>j$, then there are no nontrivial maps from $P(i)^{*}$-modules to free $P(j)^{*}$-modules. We also note that there is no possibility that $d_{t}\left(v_{k} x\right)=v_{i} y$ for $x \in \tilde{K}_{s}$ and $y \in E_{t}^{o d d, *}, t<2 p^{j}-1$. Indeed there is the map $i^{*}$ of spectral sequences from that for $B P^{*}(X)$ to that for $P(i)^{*}(X)$; in the last spectral sequence $E_{2 p^{i}-1}^{* * *} \cong P(i)^{*} \otimes H^{*}(X ; \mathbb{Z} / p)$ and $i^{*}\left(v_{i} y\right) \neq 0$. Hence the next nonzero differential must be of the form $d_{2 p^{i}-1}(x)=v_{i} \otimes Q_{i}(x)$. Therefore we have

$$
E_{2 p^{i}}^{*, *} \cong C(i+1) \oplus D(i) \oplus P(i+1) Q_{i} \ldots Q_{0} G_{i} \oplus B P^{*}\left\{p, \ldots, v_{i-1}\right\} \tilde{K}_{i-1} .
$$

The last term is computed from $Q_{i} \tilde{K}_{i-1} \neq 0$ and $\operatorname{Ker} d_{2 p^{i}-1} \mid B P^{*}\left\{\tilde{K}_{i-1}\right\}=$ $B P^{*}\left\{p, \ldots, v_{i-1}\right\} \tilde{K}_{i-1}$, since $Q_{i} \tilde{K}_{i-1}$ is $P(i)^{*}$-free in $E_{2 p^{i}-1}^{*, *}$. 
The classifying spaces of groups $B O(n), S O(4), G_{2}, \operatorname{Spin}(m), m \leq 9$ for $p=2$ and $P G L_{3}, F_{4}$ for $p=3$, and $(\mathbb{Z} / p)^{n}$ satisfy the assumption of the above lemma. However $S O(6)$ does not satisfy the above lemma [I].

We will show that the isomorphism (1) in Lemma 5.2 approximates $h^{* * *}(X ; \mathbb{Z} / p)$. Let $I h^{*, *}(X)$ be a $\mathbb{Z} / p[\tau]$-submodule of $h^{*, *}(X ; \mathbb{Z} / p)$ generated by image from $h^{*, *}(X) / p$. The following theorem is almost immediate.

Theorem 5.3. Suppose that (1) in Lemma 5.2 holds. Then we have the injection

$$
\begin{gathered}
I h^{*, *} \hookrightarrow\left(\left(G_{-1} / p \bigoplus_{n=1} Q(1, n) G_{n}^{\prime}\right)-\left(\bigoplus_{s=1} Q(1, s) K_{s}^{\prime}-\tilde{K}_{s}^{\prime}\right)\right) \otimes \mathbb{Z} / p[\tau], \\
h^{*, *}(X ; \mathbb{Z} / p) \hookrightarrow\left(\bigoplus_{n=1} Q(n) G_{n}-\left(\bigoplus_{s=1} Q(s) K_{s}-\tilde{K}_{s}\right)\right) \otimes \mathbb{Z} / p[\tau],
\end{gathered}
$$

with $w\left(G_{n}\right)=n+1, w\left(G_{n}^{\prime}\right)=n$. Moreover, if some $B P^{*}$-module generator in $\operatorname{Ideal}\left(p, . ., v_{1}\right) \tilde{K}_{s} \subset E_{\infty}^{* * *}$ is represented by transfer of a Chern class, then $\operatorname{Ker}\left(t_{\mathbb{C}}^{2 * * *}\right)$ contains a nonzero element.

The $P(m)^{*}(-)$ version of above facts also holds, if we consider the spectral sequence

$$
E_{2}^{*, *}=H^{*}(X ; \mathbb{Z} / p) \otimes P(m)^{*} \Longrightarrow P(m)^{*}(X) .
$$

$(5.3)^{\prime} \quad$ Let $E_{\infty}^{*, *}=A \oplus B$, where $A$ (resp. $B$ ) is the $P(m)^{*}$-module generated by generators in $E_{\infty}^{*, 0}$ (resp. in $E_{\infty}^{*, \text { minus }}$ ).

Lemma 5.4. (1) If (5.2) is satisfied and, in (5.3)',

$$
A \cong \bigoplus_{n=-1} P(m+n+1)^{*} \tilde{G}_{n}(m), \quad B \cong \bigoplus_{s=0} P(m)^{*}\left\{v_{m}, \ldots, v_{s}\right\} K_{s}(m),
$$

then we have the isomorphism

$$
\left.H^{*}(X ; \mathbb{Z} / p) \cong\left(\bigoplus_{n=-1} Q(m, n+m) G_{n}(m)\right)-\bigoplus_{s=0} Q(m, m+s) K_{s}(m)-\tilde{K}_{s}(m)\right)
$$

with $Q_{m} \ldots Q_{m+n} G_{n}(m)=\tilde{G}_{n}(m)$ and $Q_{m} \ldots Q_{m+s} K_{s}(m)=\tilde{K}_{s}(m)$.

(2) If $Q_{m} \ldots Q_{m+n} G_{n}(m) \in \operatorname{Im}(\rho)$ and $\left|\tilde{K}_{s}(m)\right|=$ even, then the converse also holds.

The $P(m)^{*}$-versions also hold for $G=(\mathbb{Z} / p)^{n}, B O(n), B S O(4), p_{+}^{1+2}$. One application for the above lemma is the following.

Corollary 5.5. Let $H^{*}(X ; \mathbb{Z} / p)$ (resp. $H^{*}(Y ; \mathbb{Z} / p)$ ) have the decomposition of Lemma 5.2 (1) (resp. Lemma 5.4 (1) for all $m \geq 0$ ). Then $H^{*}(X \times Y ; \mathbb{Z} / p)$ also has decomposition similar to that of Lemma 5.2 (1).

Proof. We get the following isomorphism:

$$
\begin{gathered}
Q(n-1) G_{n-1} \otimes H^{*}(Y ; \mathbb{Z} / p) \\
\left.\cong Q(n-1) G_{n-1} \otimes\left(Q(n, n+k) G_{k}(n)-\bigoplus Q(n, n+t) K_{t}(n)-\tilde{K}_{t}(n)\right)\right) \\
\cong\left(Q(n+k) G_{n-1} \otimes G_{k}(n)\right)-\left(Q(n+t) G_{n-1} \otimes K_{t}(n)-Q(n-1) G_{n-1} \otimes \tilde{K}_{s}(n)\right),
\end{gathered}
$$
since each $Q_{i}$ is derivative.

Lemma 5.6. If $H^{*}(X ; \mathbb{Z} / p) \cong \bigoplus Q(n) G_{n}$, then $H^{*}(X \times B \mathbb{Z} / p) \cong \bigoplus Q(n) G_{n}^{\prime}$, where

$$
G_{n}^{\prime} \cong \mathbb{Z} / p[y] /\left(y^{p^{n}}\right) G_{n} \oplus \mathbb{Z} / p[y] G_{n-1}\{x\} .
$$


Proof. Since we have the decomposition

$$
H^{*}(B \mathbb{Z} / p ; \mathbb{Z} / p) \cong \mathbb{Z} / p[y] /\left(y^{p^{n}}\right) \oplus \mathbb{Z} / p[y] Q(n, n)\{x\},
$$

we get the lemma.

When $X=(B \mathbb{Z} / p)^{n}$, inductively we get the decomposition $H^{*}\left((B \mathbb{Z} / p)^{n} ; \mathbb{Z} / p\right) \cong$ $\bigoplus Q(n) G_{n}$. Hence $B=0$ and

$$
g r B P^{*}(X) \cong \bigoplus P(n+1)^{*} \tilde{G}_{n}, \quad H^{*, *}(X ; \mathbb{Z} / p) \cong \bigoplus Q(n) G_{n} \otimes \mathbb{Z} / p[\tau] .
$$

Of course these are given in (3.9). The similar facts also hold for $X=B O(n)$. Moreover, W. S. Wilson proved [RWY that

$$
B P^{*}(B O(n)) \cong B P^{*}\left[c_{1}, \ldots, c_{n}\right] /\left(c_{1}-c_{1}^{*}, \ldots, c_{n}-c_{n}^{*}\right),
$$

where $c_{i}^{*}$ is the complex conjugate of the Chern class of the usual complex representation. The cohomology $h^{*, *}(B O(n))$ is studied in (4.2).

Next consider the case $X=B S O(4)$. The mod 2 cohomology is $H^{*}(X ; \mathbb{Z} / 2) \cong$ $\mathbb{Z} / 2\left[w_{2}, w_{3}, w_{4}\right]$. The cohomology operation acts as

$$
Q_{0} w_{2}=w_{3}, Q_{1} w_{3}=w_{3}^{2}, Q_{1} w_{4}=w_{4} w_{3}, Q_{1} Q_{2} w_{4}=w_{3}^{2} w_{4}^{2} .
$$

The integral cohomology is written as

$$
H^{*}(X)_{(2)} \cong Z_{(2)}\left[w_{2}^{2}, w_{4}\right] \otimes\left(Z_{(2)}\{1\} \oplus \mathbb{Z} / 2\left[w_{3}\right]\left\{w_{3}\right\}\right) .
$$

In the Atiyah-Hirzebruch spectral sequence, nonzero differentials are $d_{2^{i+1}-1}(x)=$ $v_{i} \otimes Q_{i}(x)$ for $i=1,2$. We can compute

$$
\begin{gathered}
E_{\infty}^{* * *} \cong E_{8}^{*, *} \cong Z_{(2)}\left[c_{2}\right] \otimes\left(B P^{*}\left[c_{4}\right]\left\{1,2 w_{4}\right\} \oplus P(2)^{*}\left[c_{3}\right]\left\{c_{3}\right\} \oplus P(3)^{*}\left[c_{3}, c_{4}\right]\left\{c_{3} c_{4}\right\}\right), \\
B P^{*}(X) \otimes_{B P^{*}} Z_{(2)} \cong Z_{(2)}\left[c_{2}, c_{4}\right] \otimes\left(Z_{(2)}\left\{1,2 w_{4}\right\} \oplus \mathbb{Z} / 2\left[c_{3}\right]\left\{c_{3}\right\}\right) .
\end{gathered}
$$

Hence the assumption of (1) in Lemma 5.2 is satisfied by

$$
\begin{gathered}
\tilde{G}_{-1}^{\prime} \cong \mathbb{Z} / 2\left[c_{2}, c_{4}\right], \quad \tilde{G}_{1}^{\prime}=\mathbb{Z} / 2\left[c_{2}, c_{3}\right]\left\{c_{3}\right\} \quad \tilde{G}_{2}^{\prime}=\mathbb{Z} / 2\left[c_{2}, c_{3}, c_{4}\right]\left\{c_{3} c_{4}\right\}, \\
\tilde{K}_{0}^{\prime}=\mathbb{Z} / 2\left[c_{2}, c_{4}\right]\left\{2 w_{4}\right\} .
\end{gathered}
$$

Therefore we get

Proposition 5.7. Let $w\left(w_{4}\right)=2$. Then the bidegree $\mathbb{Z} / 2[\tau]$-module $I h^{*, *}(B S O(4))$ (resp. $\left.h^{* * *}(B S O(4) ; \mathbb{Z} / 2)\right)$ is isomorphic to a bidegree $\mathbb{Z} / 2[\tau]$ - submodule of

$$
\left.\mathbb{Z} / 2\left[\tau, c_{2}\right] \otimes\left(\mathbb{Z} / 2\left[c_{4}\right]\{1\} \oplus \mathbb{Z} / 2\left[c_{3}\right] \otimes Q(1,1)\left\{w_{3}\right\} \oplus \mathbb{Z} / 2\left[c_{3}, c_{4}\right] \otimes Q(1,2)\left\{w_{4}\right\}\right)\right)
$$

(resp. $\mathbb{Z} / 2\left[\tau, c_{2}\right] \otimes\left(\mathbb{Z} / 2\left[c_{4}\right]\{1\} \oplus \mathbb{Z} / 2\left[c_{3}\right] \otimes Q(1)\left\{w_{2}\right\} \oplus \mathbb{Z} / 2\left[c_{3}, c_{4}\right] \otimes(Q(2)-\mathbb{Z} / p)\{a\}\right)$, where $Q_{0} a=w_{4}$.

Remark. If $w_{4} \in H^{4,3}(B S O(4))$, then $I^{*, *}(B S O(4))$ is isomorphic to the $\mathbb{Z} / 2[\tau]$ module in the above proposition.

Remark. For this case, we have $K_{0}=\mathbb{Z} / 2\left[c_{2}\right]\{a\}$ and $Q_{0} K_{0}=K^{\prime}{ }_{0}$ in Lemma 5.2. Indeed, $Q_{0} a=w_{4}$. However, $w_{4} \notin \operatorname{Im}\left(Q_{0}\right)$ in $h^{*, *}(B S O(4) ; \mathbb{Z} / 2)$, because $a$ itself does not exist in $h^{*, *}(B S O(4) ; \mathbb{Z} / 2)$. 
We know that the element corresponding to $2 w_{4}$ is represented by a Chern class $c_{2}^{\prime}$ of some representation, and this means the Totaro's cycle map $\tilde{c l}$ is epic. Indeed, Totaro and Pandharipande showed that this map is isomorphic, namely,

$$
C H^{*}(B S O(4))_{(2)} \cong Z_{(2)}\left[c_{2}, c_{3}, c_{4}, c_{2}^{\prime}\right] /\left(2 c_{3}, c_{3} c_{2}^{\prime}, c_{2}^{\prime 2}-4 c_{4}\right) .
$$

Next consider the $P(1)^{*}$-version for $B S O(4)$. By using the computations of $Q_{i} w_{j}$ [I] and the Atiyah-Hirzebruch spectral sequence, we can prove that

$$
\begin{gathered}
\operatorname{gr} P(1)^{*}(B S O(4)) \cong P(1)^{*}\left[c_{4}\right]\left\{1, v_{1} w_{2} w_{4}\right\} \oplus P(2)^{*}\left\{c_{3}\right\} \\
\oplus P(3)^{*}\left[c_{3}\right]\left\{c_{3}^{2}, c_{3} c_{4}\right\} \oplus P(3)^{*}\left[c_{4}\right]\left\{c_{3} c_{4}^{2}\right\} \oplus P(4)^{*}\left[c_{3}, c_{4}\right]\left\{c_{3}^{2} c_{4}^{2}\right\}
\end{gathered}
$$

We have another decomposition of $H^{*}(B S O(4) ; \mathbb{Z} / 2)$.

\section{Proposition 5.8.}

$$
\begin{gathered}
H^{*}(B S O(4) ; \mathbb{Z} / 2) \cong \mathbb{Z} / 2\left[c_{4}\right] \oplus Q(1,1)\left\{w_{3}\right\} \oplus \mathbb{Z} / 2\left[c_{3}\right] \otimes\left(Q(1,2)\left\{w_{2}, w_{4}\right\}\right) \\
\oplus \mathbb{Z} / 2\left[c_{4}\right] \otimes\left(Q(1,2)\left\{c_{4} w_{4}\right\}\right) \oplus \mathbb{Z} / 2\left[c_{3}, c_{4}\right] \otimes\left(Q(1,3)\left\{Q_{1}^{-1} w_{2} w_{4}\right\}-\left\{Q_{1}^{-1} w_{2} w_{4}\right\}\right) .
\end{gathered}
$$

We consider the relation between $\operatorname{gr} B P^{*}(X)$ and $\operatorname{gr} P(1)^{*}(X)$. When $X=$ $B S O(4)$, it is known $\left[\mathrm{KY}\right.$ that $K(n)^{\text {odd }}(X)=0$, and hence

$$
P(m)^{*}(X) \cong P(m)^{*} \otimes_{B P^{*}} B P^{*}(X) .
$$

Therefore no $P(m)^{*}(X)$ is $v_{m}$-torsion. Of course we have already seen that for the $\operatorname{gr} B P^{*}(-)$-versions the above facts do not hold. If there is a relation $p a_{0}+v_{1} a_{1}+$ $v_{2} a_{2}+\ldots=0 \in B P^{*}(X)$, then it is known $\mathrm{Y1}$ that there is $y \in H^{*}(X ; \mathbb{Z} / p)$ such that $Q_{i}(y)=\rho\left(a_{i}\right)$, where $\rho: B P^{*}(X) \rightarrow H^{*}(X ; \mathbb{Z} / p)$ is the Thom map. In $H^{*}(B S O(4) ; \mathbb{Z} / 2)$, we see that

$$
Q_{0}\left(w_{2} w_{3}\right)=c_{3}, Q_{1}\left(w_{2} w_{3}\right)=0, Q_{2}\left(w_{2} w_{3}\right)=c_{3}^{2} .
$$

Hence we have the relation $2 c_{3}+v_{2} c_{3}^{2}+\ldots=0 \in B P^{*}(B S O(2))$. This shows that $c_{3}^{2}$ is $P(2)^{*}$-free in $\operatorname{gr} B P^{*}(B S O(4))$, but it is a $P(3)^{*}$-free module in

$$
\operatorname{gr} P(1)^{*}(B S O(4))=\operatorname{gr}\left(B P^{*}(B S O(4)) / 2\right) .
$$

We also see that for $x=c_{3} w_{3} w_{4}+c_{4} w_{2} w_{3}$

$$
Q_{0}(x)=c_{3} c_{4}, \quad Q_{1}(x)=Q_{2}(x)=0, \quad Q_{3}(x)=c_{3}^{2} c_{4}^{2} .
$$

This means that $2 c_{3} c_{4}+v_{3} c_{3}^{2} c_{4}^{2}+\ldots=0 \in B P^{*}(B S O(4))$. Hence $c_{3}^{2} c_{4}^{2}$ is a $P(3)^{*}$-free module in $\operatorname{gr} B P^{*}(B S O(4))$ but is a $P(4)^{*}$-free module in $\operatorname{gr}\left(B P^{*}(B S O(4)) / 2\right)$.

Next consider the case $X=B S O(6)$. In this case the assumption (5.3) is not satisfied. In fact, Inoue computed [I]

$$
\operatorname{grBP} P^{*}(B S O(6)) \cong \bigoplus_{n=-1}^{4} P(n+1)^{*} \tilde{G}_{n} \oplus P(2)^{*} /\left(v_{2}^{2}\right) \tilde{G}_{1}^{\prime} \oplus B P^{*}\{2\} \tilde{K}_{0} .
$$

(For details, see [].) In particular, he showed that

$$
d_{5}\left(2 w_{6}\right)=v_{1}^{2} w_{6} w_{5}, \quad d_{11}\left(v_{1} \otimes w_{6} w_{5}\right)=v_{2}^{2} w_{6}^{2} w_{5}^{2} .
$$

However, even this case we can show that

$$
H^{*}(B S O(6) ; \mathbb{Z} / 2) \subset \bigoplus Q(n) G_{n} \oplus Q(1) G_{1}^{\prime} .
$$

Moreover, R. Field [F] announced that

$$
C H^{*}(B S O(2 n)) \cong Z_{(2)}\left[c_{2}, \ldots, c_{2 n}, y_{n}\right] /\left(2 c_{o d d}, c_{o d d} y_{n}, y_{n}^{2}-(-1)^{n} 2^{2 n-2} c_{2 n}\right)
$$


with $\operatorname{deg}\left(y_{n}\right)=2 n$. Hence $\operatorname{Ideal}\left(y_{n}\right) \subset \operatorname{Ker}\left(t_{\mathbb{C}}\right)$. However, $y_{n}$ is not represented by a Chern class of any representation for $n>2$. We also note that $B P^{*}(B S O(2 n))$ are not known for $n>3$.

The cases $X=B G_{2}, B \operatorname{Spin}(7)$ are quite similar to the case $X=B S O(4)$. Indeed, $C H^{*}\left(B G_{2}\right) / 2$ and $h^{* * *}\left(B G_{2} ; \mathbb{Z} / 2\right)$ have been discussed in $\S 4$, and

$$
\operatorname{gr} B P^{*}\left(B G_{2}\right) \cong Z_{(2)}\left[c_{4}, c_{6}\right] \otimes\left(B P^{*}\left\{1,2 w_{4}\right\} \oplus P(3)^{*}\left[c_{7}\right]\left\{c_{7}\right\}\right) .
$$

The infinite term of the spectral sequence for $B P^{*}(B \operatorname{Spin}(7))$ is computed by

$$
\begin{aligned}
& Z_{(2)}\left[c_{4}, c_{6}\right] \otimes\left(B P^{*}\left[c_{8}\right]\left\{1,2 w_{4}, 2 w_{8}, 2 w_{4} w_{8}, v_{1} w_{8}\right\}\right. \\
&\left.\oplus P(3)^{*}\left[c_{7}\right]\left\{c_{7}\right\} \oplus P(4)^{*}\left[c_{7}, c_{8}\right]\left\{c_{7} c_{8}\right\}\right) .
\end{aligned}
$$

Therefore we obtain

Corollary 5.9. Let $w\left(w_{8}\right)=2$. Then the cohomology $I h^{*, *}(B \operatorname{Spin}(7))$ (resp. $h^{*, *}(B \operatorname{Spin}(7) ; \mathbb{Z} / 2)$ ) is isomorphic to a $\mathbb{Z} / 2[\tau]$-submodule of $\mathbb{Z} / 2\left[\tau, c_{4}, c_{6}\right] \otimes A$ (resp. $\left.\mathbb{Z} / 2\left[\tau, c_{4}, c_{6}\right] \otimes B\right)$, where

$$
\begin{aligned}
& A=\mathbb{Z} / 2\left[c_{8}\right] \oplus \mathbb{Z} / 2\left[c_{7}\right] \otimes Q(1,2)\left\{w_{4}\right\} \oplus \mathbb{Z} / 2\left[c_{7}, c_{8}\right] \otimes(Q(1,3)-\mathbb{Z} / p)\{b\}, \\
& B=\left(\mathbb{Z} / 2\left[c_{8}\right] \oplus \mathbb{Z} / 2\left[c_{7}\right](Q(2)-\mathbb{Z} / p)\{a\} \oplus \mathbb{Z} / 2\left[c_{7}, c_{8}\right]\left(Q(3)-Q(1)+Q_{0} Q_{1}-Q_{2}\right)\{c\}\right.
\end{aligned}
$$

with $Q_{1} b=w_{8}, Q_{0} a=w_{4}, Q_{1} Q_{0} c=w_{8}, Q_{2} Q_{0} c=w_{4} w_{8}$.

The algebra $B P^{*}(B \operatorname{Spin}(7)) \otimes_{B P^{*}} Z_{(2)}$ is isomorphic to

$$
Z_{(2)}\left[c_{4}, c_{6}, c_{8}\right] \otimes\left(Z_{(2)}\left\{1,2 w_{4}, 2 w_{8}, 2 w_{4} w_{8}\right\} \oplus \mathbb{Z} / 2\left\{v_{1} w_{8}\right\} \oplus \mathbb{Z} / 2\left[c_{7}\right]\left\{c_{7}\right\}\right) .
$$

It is known that $2 w_{2}, 2 w_{8}, 2 w_{4} w_{8}$ are represented by Chern classes but $v_{1} w_{8}$ is not. However, Totaro has shown that the cycle map $\tilde{c l}$ is epic for this case also (see $\mathrm{ScY}],[\mathrm{Y} 3]$ ).

Corollary 5.10. There is an epimorphism

$$
C H^{*}(B \operatorname{Spin}(7)) \rightarrow Z_{(2)}\left[c_{4}, c_{6}, c_{8}^{\prime}\right] \otimes\left(Z_{(2)}\left\{1, c_{2}^{\prime}, c_{4}^{\prime}, c_{6}^{\prime}\right\} \oplus \mathbb{Z} / 2\left\{\xi_{3}\right\} \oplus \mathbb{Z} / 2\left[c_{7}\right]\left\{c_{7}\right\}\right),
$$

where $c_{i}^{\prime}$ is the $i$-th Chern class of complexification of the spin representation $\Delta$ and $\xi_{3}$ is a 6-dimensional element which is not represented by Chern classes. Thus $c_{2}^{\prime}, c_{4}^{\prime}, c_{6}^{\prime}$ are in $\operatorname{Ker}\left(\rho_{2}\right)$ and $\xi_{3} \in \operatorname{Ker}(\rho)$.

Next we consider the case $p=o d d$. The cases $P G L_{3}$ and $p_{+}^{1+2}$ are easy, and $I h^{* * *}(B G)$ are given. For example, for $E=p_{+}^{1+2}$

$$
\operatorname{gr} B P^{*}(B E) \cong B P^{*} \otimes H^{\text {even }}(B E) /\left(v_{1} Q_{1} H^{\text {odd }}(B E)\right) .
$$

Finally we consider the case $G=F_{4}, p=3$, whose Chow ring is still unknown. The mod 3 cohomology of $F_{4}$ is isomorphic to $H^{*}\left(B F_{4} ; \mathbb{Z} / 3\right) \cong C \otimes D$ [Tod] with $D=Z_{(3)}\left[x_{36}, x_{48}\right]$ and

$$
C=\mathbb{Z} / 3\left[x_{4}, x_{8}\right] \otimes\left\{1, x_{20}, x_{20}^{2}\right\} \oplus \mathbb{Z} / 3\left[x_{26}\right] \otimes \Lambda\left(x_{9}\right) \otimes\left\{1, x_{20}, x_{21}, x_{25}\right\},
$$

where two terms of $C$ have the intersection $\left\{1, x_{20}\right\}$. Then we can prove $[\mathrm{KY}$

$$
\operatorname{gr} B P^{*}\left(B F_{4}\right) \cong D \otimes\left(B P^{*}\left\{1,3 x_{4}\right\} \oplus B P^{*} \otimes E \oplus P(3)^{*}\left[x_{26}\right]\left\{x_{26}\right\}\right)
$$

with $E=Z_{(3)}\left[x_{4}, x_{8}\right]\left\{a b \mid a, b \in\left\{x_{4}, x_{8}, x_{20}\right\}\right\}$. Therefore we obtain

Corollary 5.11. Let $w(E)=0$ and $w\left(x_{4}\right)=2$. Then $I^{*, *}\left(B F_{4}\right)$ is a $\mathbb{Z} / 3[\tau]$ submodule of

$$
\left.D \otimes\left(\mathbb{Z} / 3\{1\} \oplus E \oplus \mathbb{Z} / 3\left[x_{26}\right] \otimes Q(1,2)\left\{x_{4}\right\}\right)\right) \otimes \mathbb{Z} / 3[\tau] .
$$


The element $3 x_{4}$ can be proved to be represented by a Chern class, and $x_{26}=$ $Q_{2} Q_{1} x_{4}$. The element $x_{36}$ is also represented by a Chern class, and $P^{3} x_{36}=x_{48}$. If we can prove that $E / 3 \subset \operatorname{Im}\left(c l_{p}\right)$ and $x \in H^{4,3}\left(B F_{4}, \mathbb{Z} / 3\right)$, then the above module is just $I h^{* * *}\left(B F_{4}\right)$ for $p=3$.

Let $G$ be a simply connected Lie group. Then $H^{3}(G ; \mathbb{Z}) \cong \mathbb{Z}$ and $H^{4}(G ; \mathbb{Z}) \cong 0$. Suppose that $H^{*}(G ; \mathbb{Z})$ has $p$-torsion. Then it is known that there is an element $x^{\prime} \in H^{3}(G ; \mathbb{Z})$ such that $0 \neq Q_{1} x^{\prime} \in H^{2 p+2}(G ; \mathbb{Z} / p)$. Taking the classifying space, we get an element $x \in H^{4}(B G ; Z)$ such that $Q_{1} x \neq 0$ in $H^{2 p+3}(B G ; \mathbb{Z} / p)$. By Totaro To2] it is known that $C H^{*}(B G) \otimes \mathbb{Q} \cong H^{*}(B G) \otimes \mathbb{Q}$. Hence there is an $s \geq 1$ such that $p^{s} x_{4} \in H^{4}(B G)$ is in $\operatorname{Im}(c l)$. Thus there is a nonzero element $c \in C H^{2}(B G) / p$ with $t_{\mathbb{C}}^{2 * * *}(c)=0$. For the groups $G_{2}$ or $\operatorname{Spin}(7)$ for $p=2$ and $G=F_{4}$ for $p=3$, we can take $s=1$, since $p x_{4}$ is represented by the second Chern class $c_{2}$.

Proposition 5.12. Let $p=2,3$ or 5 . There is a classifying space $B \tilde{G}$ such that for all $m, n$ with $3 \leq n+1<m \leq 2 n$, the kernel $\operatorname{Ker}\left(t_{\mathbb{C}}^{m, n}\right)$ is nonzero.

Proof. Let $\tilde{G}=G \times(\mathbb{Z} / p)^{\infty}$, where $G=G_{2}, p=2, G=F_{4}, p=3$ or $G=E_{8}, p=5$. Recall that $(B \mathbb{Z} / p)^{n}$ satisfies the Künneth formula for all spaces. For $\mathbb{Z} / p[\tau]$ module generators $x \in H^{*, *}\left((B \mathbb{Z} / p)^{\infty} ; \mathbb{Z} / p\right)$, the elements $x c$ are all nonzero and all in Ker $t_{\mathbb{C}}$.

\section{Homotopy CATEGory}

From the category $S p c$, Voevodsky constructed [Vo1], [Vo2], $\mathrm{MoV0}$ ] the $\left(\mathbb{A}^{1}\right.$, algebraic) homotopy category $H o t$ and the stable homotopy category SHot. There are two different types of spheres in $S p c$ :

$$
S_{s}^{1}=\mathbb{A}^{1} /\{0,1\} \quad \text { and } \quad S_{t}^{1}=\mathbb{A}^{1}-\{0\} .
$$

The Tate object is $T=\mathbb{A}^{1} /\left(\mathbb{A}^{1}-0\right) \cong \mathbb{P}^{1} \cong S_{t}^{1} \wedge S_{s}^{1}$ in Hot. The category SHot is defined by $T$ as the suspension, e.g., $E=\left\{E_{i}\right\}, E_{i} \in S p t$ is a spectrum if there is a map $T \wedge E_{i} \rightarrow E_{i+1}$.

Let $\Sigma_{T}^{\infty}$ be the functor from $S p c$ to $T$-spectra that takes $X$ to $\left\{T^{i} \wedge X\right\}$. If $E$ is a $T$-spectrum, then the motivic (generalized) cohomology $E^{*, *}(-)$ is defined by

$$
\begin{aligned}
& E^{m, n}(X)=\operatorname{Hom}_{S H o t}\left(\Sigma_{T}^{\infty}(X), S_{s}^{m-n} \wedge S_{t}^{n} \wedge E\right), \\
& E_{m, n}(X)=\operatorname{Hom}_{S H o t}\left(S_{s}^{m-n} \wedge S_{t}^{n}, \Sigma_{T}^{\infty}(X) \wedge E\right),
\end{aligned}
$$

where $\operatorname{Hom}_{S H o t}(-,-)$ is the homomorphism defined on $S H o t$.

The realization map $t_{\mathbb{C}}$ is originally defined as the functor $t_{\mathbb{C}}: X \rightarrow X(\mathbb{C})$ from $H o t$ to the category of homotopy spaces. Note that this induces

$$
t_{\mathbb{C}}: E^{m, n}(X) \rightarrow\left(t_{\mathbb{C}} E\right)^{m}(X(\mathbb{C})) .
$$

The spectrum for the ordinary motivic cohomology is defined as follows. Let $L(X ; R)$ for $R=\mathbb{Z}$ or $\mathbb{Z} / p$ be the presheaf sending a connected $U$ to the free $R$-module generated by the set of all closed irreducible $W \subset U \times X$ such that the projection $W \rightarrow U$ is finite and surjective. The Eilenberg-MacLane spectrum is defined as

$$
K(R(n), 2 n)=L\left(\mathbb{A}^{n} ; R\right) / L\left(\mathbb{A}^{n}-\{0\} ; R\right)
$$


Voevodsky proved that $K(R(n), 2 n)$ is the $\Omega$-spectrum for the suspension $T$, namely, $K(R(n), 2 n) \cong \Omega_{T} K(R(n+1), 2 n+2)$ in Hot. Define also, for $m<2 n$,

$$
K(R(n), m)=\Omega_{S^{1}}^{2 n-m}(R(n), 2 n) .
$$

Thus the ordinary motivic cohomology is defined by

$$
H^{m, n}(X ; R)=\operatorname{Hom}_{H o t}(X, K(R(n), m)) .
$$

Question 6.1. Let $k \subset \mathbb{C}$, and let $0 \neq \tau_{n} \in H^{n, n}(K(\mathbb{Z} / p(n), n) ; \mathbb{Z} / p)$ (resp. $\left.\tau_{n+1}^{\prime} \in H^{n+1, n}\left(K\left(\mathbb{Z}_{(p)}(n), n+1\right) ; \mathbb{Z} / p\right)\right)$ be the fundamental class (representing the identity map). Then are there isomorphisms

$$
\begin{gathered}
h^{2 *, *}(K(\mathbb{Z} / p(n), n) ; \mathbb{Z} / p) \cong \mathbb{Z} / p\left[Q_{i_{n-1}} \ldots Q_{i_{1}} Q_{0} \tau_{n} \mid 0<i_{1}<\ldots<i_{n-1}\right], \\
h^{2 * * *}\left(K\left(Z_{(p)}(n), n+1\right) ; \mathbb{Z} / p\right) \cong \mathbb{Z} / p\left[Q_{i_{n-1}} \ldots Q_{i_{1}} \tau_{n+1}^{\prime} \mid 0<i_{1}<\ldots<i_{n-1}\right] ?
\end{gathered}
$$

It is well known that the dual $A_{p *}$ of the (topological) Steenrod algebra $A_{p}^{*}$ is isomorphic to $\mathbb{Z} / p\left[\xi_{1}, \ldots\right] \otimes \Lambda\left(\tau_{0}, \ldots\right),\left|\xi_{i}\right|=2\left(p^{i}-1\right),\left|\tau_{i}\right|=2 p^{i}-1$. Let $P^{J} \in$ $A_{p}^{*}$ (resp. $Q^{I} \in A_{p}^{*}$ ) be the dual of $\xi_{1}^{j_{1}} \ldots$ (resp. $\tau_{0}^{i_{0}} \ldots, i_{k}=0$ or 1 ), so that $A_{p}^{*} \cong \mathbb{Z} / p\left\{P^{J} Q^{I}\right\}$. Note that $Q^{I}= \pm Q_{0}^{i_{0}} \ldots$. Define $m(J)=\sum_{k=1} j_{k}$ and $m(I)=$ $\sum_{k=0} i_{k}$. Then it is also known [Ta] that

$$
H^{*}(K(\mathbb{Z} / p, n) ; \mathbb{Z} / p) \cong \mathbb{Z} / p\left[Q^{I} P^{J} \tau_{n} \mid m(I)+2 m(J)<n+i_{0}\right] .
$$

On the other hand, suppose that $Q^{I} P^{J} \tau_{n} \in H^{m, n}(K(\mathbb{Z} / p(n), n) ; \mathbb{Z} / p)$ for $m \geq$ $2 n$, i.e., $w\left(Q^{I} P^{J} \tau\right) \leq 0$. Since $w\left(P^{j}\right)=0$ and $w\left(Q_{i}\right)=-1$, we see that

$$
0 \geq w\left(Q^{I} P^{J} \tau_{n}\right)=n-m(I) .
$$

This implies $m(J)=0, m(I)=n$ and $i_{0} \neq 0$. Hence we know that $Q^{I} P^{J} \tau$ is the form of the ring generator of the polynomial in the above question.

Remark. Let us write the above as $A=\mathbb{Z} / p\left[Q_{i_{n-1}} \ldots Q_{i_{1}} Q_{0} \tau \mid 0<i_{1}<\ldots<i_{n-1}\right]$. By Tamanoi $\mathrm{Ta}$, the image $\rho_{p}(K(\mathbb{Z} / p, n))=A \subset H^{*}(K(\mathbb{Z} / p, n) ; \mathbb{Z} / p)$. Moreover, there is [RWY] the isomorphism $B P^{*}(K(\mathbb{Z} / p, n)) \otimes_{B P^{*}} \mathbb{Z} / p \cong A$.

\section{Algebraic Cobordism}

Let $B G L$ denote the infinite Grassmannian, the union of $G L_{N}(\infty)$ over $N$. The corresponding generalized cohomology theory is the algebraic $K$-theory. The motivic cobordism theory $M G L^{*, *}(-)$ is the generalized cohomology theory defined by the Thom spectrum $M G L=\left\{T h\left(E_{n} \rightarrow G L_{n}\right)\right\}_{n}$ identifying $\operatorname{Th}(E \oplus O) \cong$ $T \wedge T h(E)$ and $E_{n} \oplus O \rightarrow E_{n}$ for the trivial line bundle $O$. It is known (Hu-Kř́rź [HK, Vezzosi [Ve2]) that

$$
\begin{aligned}
& M G L^{*, *}\left(\left(\mathbb{P}^{\infty}\right)^{n}\right) \cong M G L^{*, *}(p t)\left[y_{1}, \ldots, y_{n}\right], \\
& \operatorname{MGL}^{*, *}\left(B G L_{n}\right) \cong M G L^{*, *}(p t)\left[c_{1}, \ldots, c_{n}\right],
\end{aligned}
$$

where the $c_{i}$ are identified with the elementary symmetric polynomials in the $y_{i}$ 's. Hence the Chern classes are also defined in $M G L^{2 * *}(B G)$. The realization maps

$$
t_{\mathbb{C}}^{2 *, *}: M G L^{2 *, *}(B G)_{(p)} \rightarrow M U^{*}(B G)_{(p)}
$$

are epic for $G=O(n), S O(4), G_{2}$ for $p=2$ and $p_{+}^{1+2}$ for all primes, because the $M U^{*}(B G)_{(p)}$ are generated by Chern classes. 
For a smooth scheme $X$ over $k \subset \mathbb{C}$, Levine and Morel [LM1, [LM2] constructed the algebraic cobordism theory $\Omega^{*}(X)$ such that there are natural maps

$$
\rho_{H}: \Omega^{*}(X) \rightarrow H^{2 *, *}(X), \quad \rho_{M G L}: \Omega^{*}(X) \rightarrow M G L^{2 *, *}(X)
$$

with $\rho_{H}=\rho_{(M G L, H)} \rho_{M G L}$ for the algebraic Thom map $\rho_{(M G L, H)}: M G L^{*, *}(X) \rightarrow$ $H^{*, *}(X)$. Moreover, they proved that

(7.4) $\rho_{H} \otimes_{\Omega^{*}} \mathbb{Z}: \Omega^{*}(X) \otimes_{\Omega^{*}} \mathbb{Z} \cong H^{2 *, *}(X), \quad t_{\mathbb{C}}^{2 * *} \rho_{M G L}: \Omega^{*}(p t) \cong M U^{2 *}(p t)$.

This implies the motivic version of the Totaro cycle map $\tilde{c} l$ :

$$
\rho_{M G L}\left(\rho_{H} \otimes_{\Omega^{*}} \mathbb{Z}\right)^{-1}: C H^{*}(X) \rightarrow M G L^{2 *, *}(X) \otimes_{M G L^{2 *, *}} \mathbb{Z},
$$

and moreover $t_{\mathbb{C}}^{2 *, *} \rho_{M G L}\left(\rho_{H} \otimes_{\Omega^{*}} \mathbb{Z}\right)^{-1}$ is the Totaro cycle map $\tilde{c l}$. Thus the Thom $\operatorname{map} \rho_{(M G L, H)}^{2 *, *}: M G L^{2 *, *}(X) \rightarrow H^{2 *, *}(X)$ is always epic.

For groups $G=(\mathbb{Z} / p)^{n}, O(n)$, we can easily prove that

$$
\Omega^{*}(B G) \cong M U^{*}(B G) .
$$

Hence in these cases $M G L^{2 * * *}(B G)$ contains $M G^{*}(B G)$ as a splitting subring.

Corollary 7.1. Let $\tilde{l}_{p}: C H^{*}(B G) / p \rightarrow M U^{*}(B G) \otimes_{M U^{*}} \mathbb{Z} / p$ be epic. Then $t_{\mathbb{C}}^{2 * * *}$ : $M G L^{2 *, *}(X) / p \rightarrow M U^{*}(B G) / p$ is epic, and $\operatorname{Im} \rho_{(M G L, h)} \subset \mathbb{Z} / p[\tau] \otimes h^{2 *, *}(X ; \mathbb{Z} / p)$, where $\rho_{(M G L, h)}: M G L^{*, *}(X) \rightarrow h^{*, *}(X ; \mathbb{Z} / p)$ is the induced Thom map.

The modified cycle maps $\tilde{c l}$ are epic also for the groups $\operatorname{Spin}(7)$ for $p=2$ and $P G L_{3}$ for $p=3$.

By the Thom isomorphism, we get $M G L^{*, *}(B G L) \cong M G L^{*, *}(M G L)$. This means that the Steenrod algebra of $M G L^{*, *}(-)$ is generated as an $M G L^{*, *}(p t)$ module by the Landweber-Novikov operation $S_{\alpha}$ :

$$
M G L^{*, *}(M G L) \cong M G L^{*, *}(p t)\left\{S_{\alpha} \mid \alpha=\left(i_{1}, \ldots, i_{n}\right), i_{j} \geq 0\right\} .
$$

Here $S_{\alpha}: M G L^{*, *}(X) \rightarrow M G L^{*+2|\alpha|, *+|\alpha|}(X)$ and $|\alpha|=\sum_{k} i_{k} k$. These operations satisfy the Cartan formula

$$
S_{\alpha}(x y)=\sum_{\alpha=\beta+\gamma} S_{\beta}(x) S_{\gamma}(y),
$$

and $S_{\alpha} \mid M U^{*}(p t)$ is the usual Landweber-Novikov operation.

Kříz, Hu and Vezzosi construct algebraic Brown-Peterson theory $A B P^{*, *}(-)$ by using a modified Quillen argument. Here we note that we can also construct algebraic BP-theory by using the technique of $\operatorname{Novikov}(5.4$ in $[\mathrm{N}])$. Recall that $M U_{(p)}^{*} \cong \mathbb{Z}_{(p)}\left[x_{1}, \ldots\right],\left|x_{i}\right|=-2 i$. Define

$$
\Delta_{x_{i}}=\sum_{q \geq 1}\left(x_{i} / S_{\Delta_{i}}\left(x_{i}\right)\right)^{q-1} S_{q \Delta_{i}}
$$

where $\Delta_{i}=(0, \ldots, 0,1,0, \ldots, 0)\left(1\right.$ in $i$-th place). Note that $\Delta_{x_{i}}\left(x_{i}\right)=S_{\Delta_{i}}\left(x_{i}\right)=1$ if $i \neq p^{j}-1$. Then we can easily prove that $\pi_{i}=1-x_{i} \Delta_{x_{i}}$ is a multiplicative projection such that $\pi_{i}\left(x_{j}\right)=\left(1-\delta_{i j}\right) x_{j}$. Essentially composing (for details, see p. 587 in $[\mathbb{N}]$ ) the $\pi_{i}$ for all $i \neq p^{j}-1$, we get the multiplicative projection $\Phi: M G L_{(p)} \rightarrow M G L_{(p)}$ such that

$$
\Phi\left(x_{i}\right)= \begin{cases}x_{i} & \left(\text { if } i=p^{j}-1 \text { for some } j\right) \\ 0 & \text { (otherwise) }\end{cases}
$$


Define the algebraic Brown-Peterson spectrum by $\Phi M G L=A B P$. Of course $t_{\mathbb{C}}(A B P)=B P$

Theorem 7.2. Identify $B P^{*}=M U_{(p)}^{*} /\left(x_{i} \mid i \neq p^{j}-1\right)$. Then

$$
A B P^{*, *}(X) \cong B P^{*} \otimes_{M U_{(p)}^{*}} M G L^{*, *}(X)_{(p)} .
$$

Proof. Since $\pi_{x_{i}}(a)=\left(1-x_{i} \Delta_{x_{i}}\right) a=a \bmod \left(x_{i}\right)$, we get $\Phi(a)=a \bmod \left(x_{i} \mid i \neq\right.$ $\left.p^{j}-1\right)$ for all $a \in M G L^{*, *}(X)$. The isomorphism is proved, since $A B P^{*, *}(X) \subset$ $M G L^{* * *}(X)_{(p)}$ by the property $\Phi^{2}=\Phi$.

Since $A B P^{*, *}(p t) \cong B P^{*} \otimes_{M U_{(p)}^{*}} M G L^{*, *}(p t)$, we can write the above isomorphism as

$$
A B P^{*, *}(X) \cong A B P^{*, *} \otimes_{M G L_{(p)}^{* * *}} M G L^{*, *}(X)_{(p)} .
$$

\section{REFERENCES}

[F] R.Field. On the Chow ring of classifying space $B S O(2 n, C)$ preprint (2000).

[GL] D. J. Green and I. J. Leary. Chern classes and extraspecial groups. Manuscripta Math. 88 (1995), 73-84. MR 96h:20096

[HK] Po Hu and I.Kř́žz. Some remarks on real and algebraic cobordism. K-theory 22 (2001), 335-366. MR 2002g:19004

[I] K.Inoue. The Brown-Peterson cohomology of BSO(6). J. Math. Kyoto Univ. 32 (1992), 655-666. MR 93k:55018

$[\mathrm{KY}] \quad$ A. Kono and N. Yagita. Brown-Peterson and ordinary cohomology theories of classifying spaces for compact Lie groups. Trans. of AMS. 339 (1993), 781-798. MR 93m:55006

[Ly] I.Leary. The integral cohomology rings of some p-groups. Math. Proc. Cambridge Philos. Soc. 110 (1991), 25-32. MR 93a:20081

[LM1] M. Levine and F. Morel. Cobordisme algébrique I. C.R.Acad.Sci.Paris Sér. I Math. 332 (2001), 723-728. MR 2002d:14031

[LM2] M. Levine and F. Morel. Cobordisme algébrique II. C.R.Acad.Sci.Paris Sér. I Math. 332 (2001) 815-820. MR 2002d:14030

[MeSu] A.Merkurjev and A.Suslin. $K$-cohomology of Severi-Brauer varieties and the norm residue homomorphism. Math. USSR Izvestiya 21 (1983), 307-340. MR 84i:12007

[MoVo] F. Morel and V. Voevodsky. $A^{1}$-homotopy theory of schemes. IHES Publ. Math. 90 (2001) 45-143. MR 2002f:14029

[N] S.P.Novikov. The methods of algebraic topology from the viewpoint of cobordism theory. Math. USSR-Izvestiya 1 (1967), 827-913. MR 36:4561

[P] R. Pandharipande. Equivariant Chow rings of $O(k), S O(2 k+1)$, and $S O(4)$. J. Reine Angew. Math. 496 (1998) 131-148. MR 99e:14001

[Q] D. Quillen. The mod 2 cohomology rings of extra-special 2-groups and the spinor groups. Math. Ann. 194 (1971), 197-212. MR 44:7582

[RWY] D. C. Ravenel, W. S. Wilson and N. Yagita. Brown-Peterson cohomology from Morava $K$-theory. K-theory 15 (1998), 147-199. MR 2000d:55012

$[\mathrm{ScY}] \quad$ B. Schuster and N. Yagita. Transfer of Chern classes in $B P$-cohomology and Chow rings. Trans. AMS.353 (2000), 1039-1054. MR 2002b:55030

[Ta] H. Tamanoi. The image of the BP Thom map for Eilenberg-MacLane spaces Trans. AMS 349 (1997) 1209-1237. MR 97i:55012

[TeY1] M. Tezuka and N.Yagita. The varieties of the mod $p$ cohomology rings of extraspecial p-groups for an odd prime p. Math. Proc. Cambridge Phil. Soc. 94 (1983) 449-459. MR 85g:20069

[TeY2] M. Tezuka and N. Yagita. Cohomology of finite groups and the Brown-Peterson cohomology. Lecture Notes in Math. 1370 (1989) 396-408. MR 90i:55011

[Tod] H.Toda. Cohomology mod 3 of the classifying space $B F_{4}$ of the exceptional group $F_{4}$. J.Math.Kyoto Univ. 13 (1973) 97-115. MR 47:9619

[To1] B.Totaro. Torsion algebraic cycles and complex cobordism. J. Amer. Math. Soc. 10 (1997), 467-493. MR 98a:14012 
[To2] B.Totaro. The Chow ring of classifying spaces. Algebraic K-theory (Seattle, 1997), Proc. Sympos. Pure Math. 67 (1999), 249-281. MR 2001f:14011

[Ve1] G.Vezzosi. On the Chow ring of the classifying stack of $P G L_{3, C}$. J.Reine Angew. Math. 523 (2000), 1-54. MR 2001f: 14012

[Ve2] G.Vezzosi. Brown-Peterson spectra in stable $A^{1}$-homotopy theory. Rend. Sem. Mat. Univ. Padova 106 (2001), 47-64. MR 2002j:55007

[Vo1] V. Voevodsky. The Milnor conjecture. Preprint (1996).

[Vo2] V. Voevodsky (Notes by C. Weibel). Voevodsky's Seattle lectures : $K$-theory and motivic cohomology. Algebraic K-theory (Seattle, 1997), Proc. Sympos. Pure Math. 67 (1999), 283-303. MR 2001i:14029

[Vo3] V. Voevodsky. Reduced power operations in motivic cohomology. preprint (2001).

[Y1] N. Yagita. On relations between Brown-Peterson cohomology and the ordinary mod $p$ cohomology theory. Kodai Math.J 7 (1984), 273-285. MR 85g:55007]

[Y2] N. Yagita. Localization of the spectral sequence converging to the cohomology of an extra special $p$-group for odd prime p. Osaka J.Math. 35 (1998), 83-116. MR 99e:20067

[Y3] N. Yagita. Chow ring of classifying spaces of extraspecial $p$ groups. Recent progress in homotopy theory, Contemp. Math. 293 (2002) 397-409.

Department of Mathematics, Faculty of Education, Ibaraki University, Mito, IbaRAKI, JAPAN

E-mail address: yagita@mx.ibaraki.ac.jp 